\title{
Mechanical behaviors of SLM additive manufactured octet-truss and truncated-octahedron lattice structures with uniform and taper beams
}

\author{
Dexing Qi ${ }^{\mathrm{a}}$, Huabin $\mathrm{Yu}^{\mathrm{a}}$, Ming Liu ${ }^{\mathrm{a}}$, Han Huang ${ }^{\mathrm{b}}$, Shucai Xu ${ }^{\mathrm{b}}$, Yong Xia ${ }^{\mathrm{b}}$, Guian Qian ${ }^{\mathrm{c}, *}$, \\ Wenwang $\mathrm{Wu}^{\mathrm{a}, \mathrm{d}, *}$
}

a Institute of Advanced Structure Technology, Beijing Institute of Technology, Beijing 100081, China

${ }^{\mathrm{b}}$ State Key Laboratory of Automotive Safety and Energy, Tsinghua University, Beijing 100084, PR China

${ }^{c}$ The State Key Laboratory of Nonlinear Mechanics, Institute of mechanics, Chinese academy of sciences, Beijing, 100190, China

${ }^{\mathrm{d}}$ Department of Mechanical Engineering, Massachusetts Institute of Technology, Cambridge, MA 02139, United States

\section{A R T I C L E I N F O}

\section{Keywords:}

Lattice structures

Homogenization theory

Mechanical properties

SLM additive manufacturing

\begin{abstract}
A B S T R A C T
In this paper, three-dimensional lattice structures composed of octet-truss and truncated-octahedron unit cells with regular and tapered beams are proposed, and their mechanical properties are investigated through experiments and simulation comparisons. Firstly, analytical closed form expressions of the relative density functions are derived for different lattice structures. Secondly, in-situ compression samples are fabricated via Selective Laser Melting (SLM) 3D printing technique, monotonic compression experiments are performed for studying the effects of tapered beams on the mechanical properties of lattice structures with ordinary uniform beams, and SEM characterizations of fracture surface morphology are performed for understanding the underlying failure mechanisms. Afterwards, asymptotic homogenization (AH) method is used for evaluating the anisotropy properties of these lattice structures, closed form expression of the mechanical behaviors of unit cells is derived and solved with ABAQUS ${ }^{\circledR}$ using surface traction method. Asymptotic homogenization analysis shows that node enforcement can significantly enhance the modulus and reduce the anisotropy of the lattice.
\end{abstract}

\section{Introduction}

Lightweight 3D Lattice structures are widely used for multifunctional applications, such as load bearing, vibration attenuation,negative and zero thermal-expansion structures, impact and blast proof structures, etc. [1-12]. According to Maxwell algebraic rule, artificial designed 2D and 3D lattices can be classified into bending-dominatedand stretchingdominated lattices $[2,3]$. Stretching-dominated lattice structures are known for their high modulus and yield strength, which makes them the best choice for lightweight structural applications, such as pyramid, Hourglass lattice, Octet and Octahedral.Stretching dominated 3D lattice normally forms two types of microscopic failure modes, namely, elastic buckling and plastic yielding. Avoiding elastic buckling failure without reaching the plastic yielding of its cell elements is important for improving the mechanical integrity of stretching-dominant 3D lattice [11].

Chen and Tan [13] derived an analytical model for predicting the effect compressive stiffness and strength of octet lattice structures with cylindrical struts, where material overlapping effects in strut joint are included. Making use of homogenization method, two approaches were employed for designing lattice structures with controlled anisotropy. One is to assemble two different base units with complementary stiff- ness along various spatial directions to forma newrepresentative unit. The other is to employ one single base unit with partially symmetrical features and array it through symmetry operations to construct a new unit which is composed of multiple original base units [14]. Meza et al. [15] investigated the strength and stiffness of solid- and hollowbeam nanolattices made of solid polymer and hollow ceramic (Al2O3), and found that their strength and stiffness are governed by an intricate combination of geometry and structural parameters. Using strain energy based homogenization methods, the macroscopic elastic stiffness tensor of BCC truss lattice materials is derived in the form of relative density, and closed-form analytical formulas are developed for bulk, Young's and shear moduli [16]. Mechanical properties of additive manufactured hollow 3D lattice structures wereinvestigated with in-situ X-ray tomography compression tests, and 3D tomography image-based conformal finite element model was performed using Gurson-Tvergaard-Needleman (GTN) porous plasticity [17]. Liu et al. [18] performed in-situ microCT interrupted quasi-static compression tests of SLS additive manufactured metallic lattices consisting of octet and rhombicuboctahedron unit cells, and statistical geometrical models on spatial distributions of defects are developed for investigating the influences of manufacturing process induced defects on the macroscopic strength degradation of the

\footnotetext{
* Corresponding authors.

E-mail addresses: qianguian@imech.ac.cn (G. Qian), wuww05@mit.edu (W. Wu).
} 
(a)
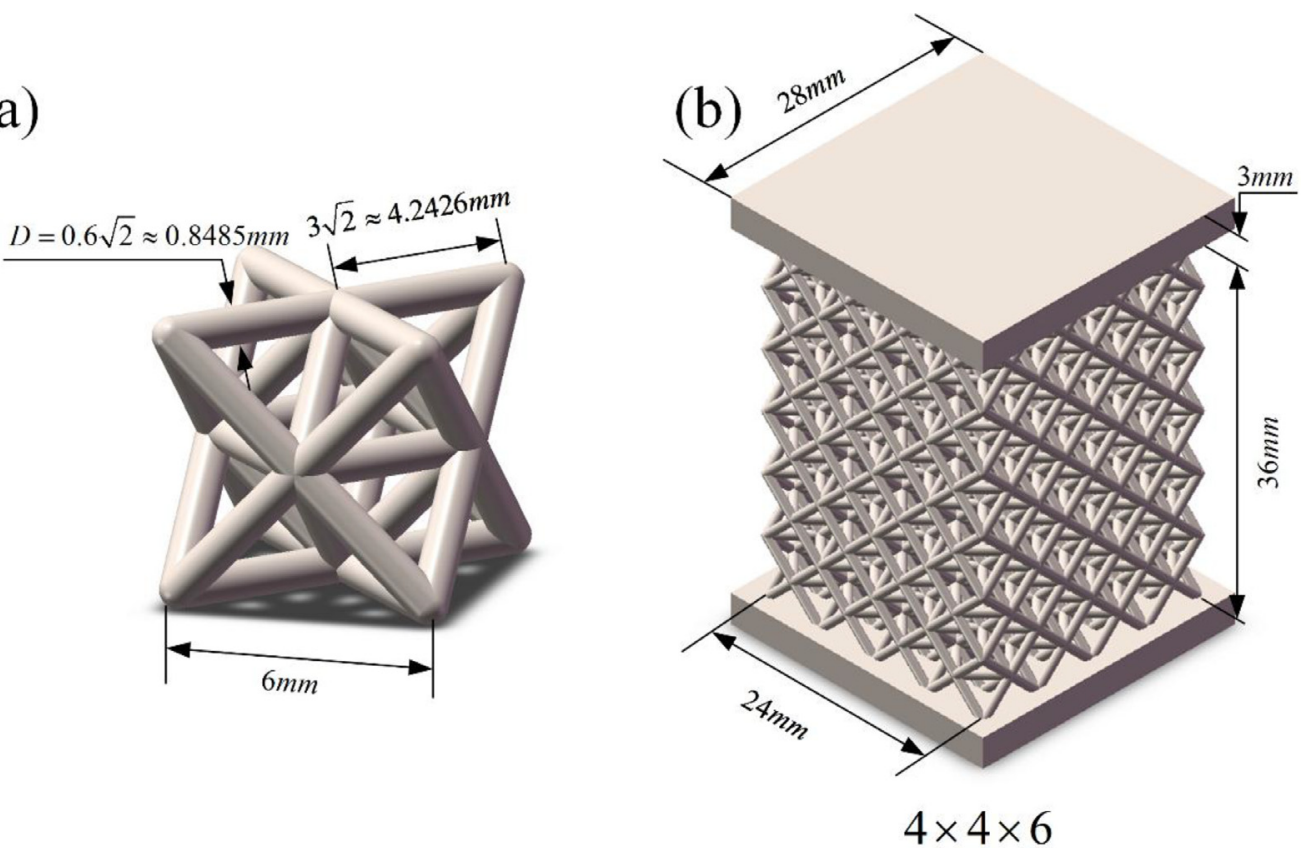

(c)

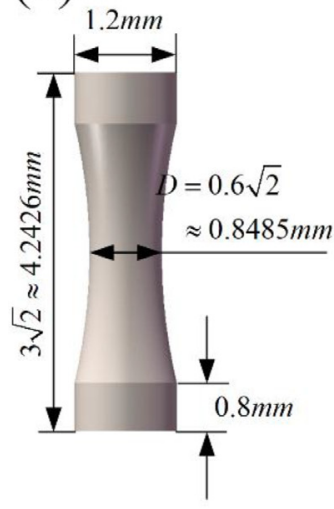

(d)

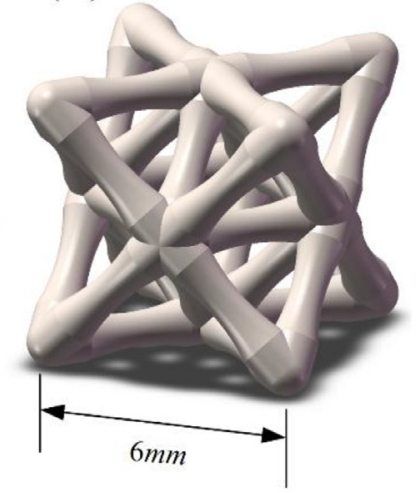

(e)

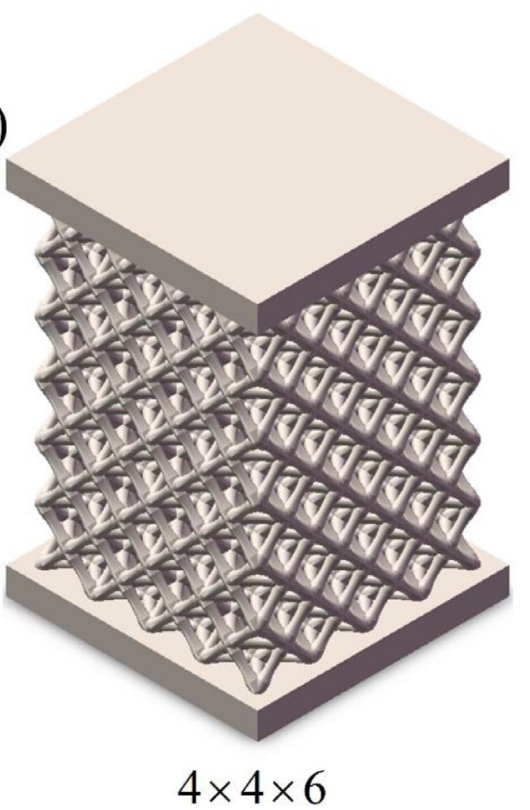

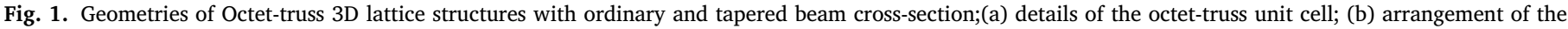

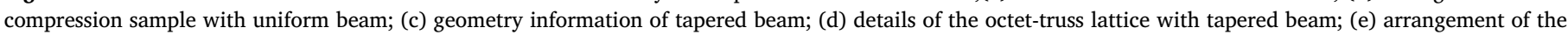
compression sample with tapered beam.

octet-truss lattice structures with different lattice angles [19]. Analytical reduced-order models including lattice node geometry features are proposed for predicting the effective strength and modulus of 3D lattice structures consisting of non-slender beams [20]. Based on the combination of classical molecular dynamics (MD) simulations and theoretical analysis, He et al. [21] investigate the mechanical properties of copper $(\mathrm{Cu})$ octet-truss nanolattices. In-situ synchrotron X-Ray microtomography uniaxial compression tests are performed on two types of 3D lattice structures, it is shown that heterogeneous localized deformation of as-fabricated lattice structures are induced bylocal lattice defects formed during manufacturing process [22]. Through experimental, theoretical and simulation verificationsand comparisons, the shearing mechanical performances of single-layer tetrahedral trusses fabricated by rapid prototyping and investment casting are investigated, and it is found that casting defects are responsible for thedrasticmechanical properties degradations of lattices manufactured with investment castingtechnique [23]. 3D space filling periodic octet-truss lattice structures from Ti-6Al-4 V alloy sheets are fabricated through combining simple snap-fit and vacuum brazing techniques, where lattice elastic stiffness constants and strengths are characterized with through-thickness compression and in-plane shearing experiments, and micromechanical models are proposed for understanding the underlying deformation and 
(a)

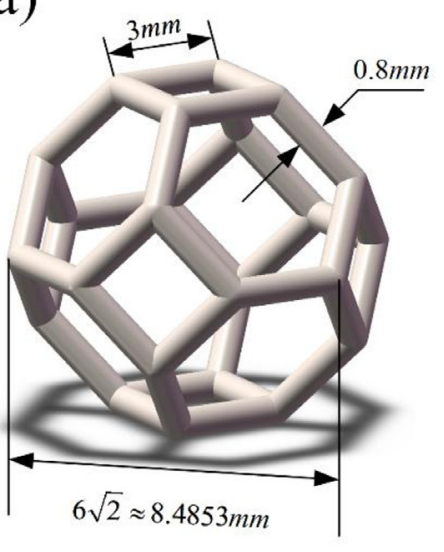

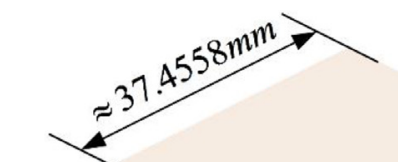

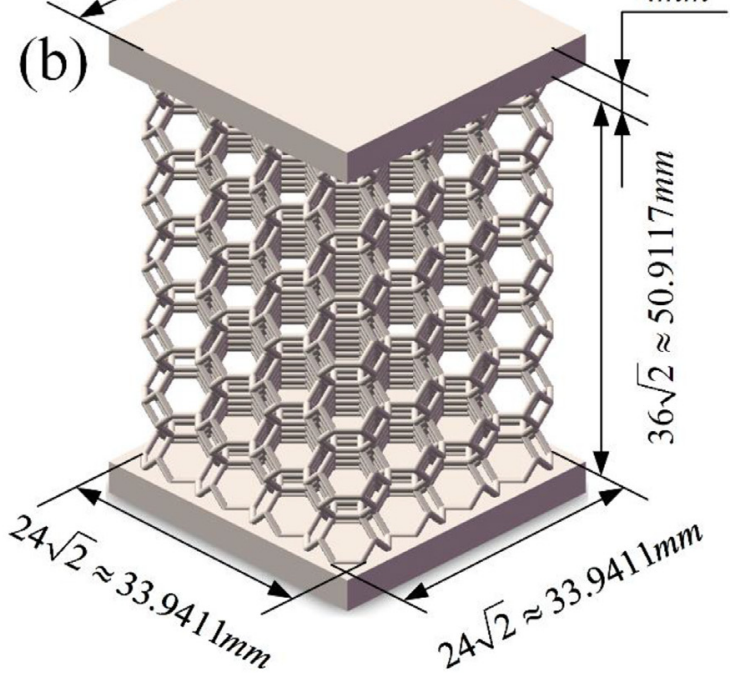

$4 \times 4 \times 6$ (c)

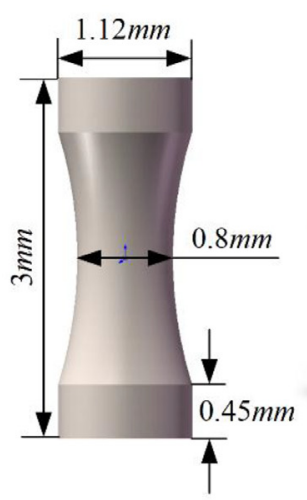

(d)

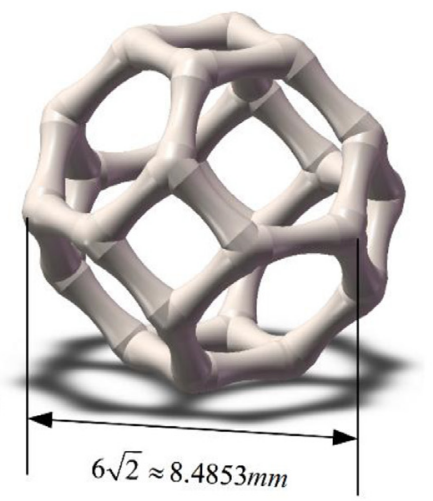

Fig. 2. Geometries of truncatedoctahedron 3D lattice structures with uniform and tapered beam cross-section; (a) details of the truncated-octahedron unit cell; (b) arrangement of the compression sample with uniform beam; (c) geometry information of tapered beam; (d) details of the tapered truncated-octahedron lattice with tapered beam; (e) arrangement of the compression sample with tapered beam.

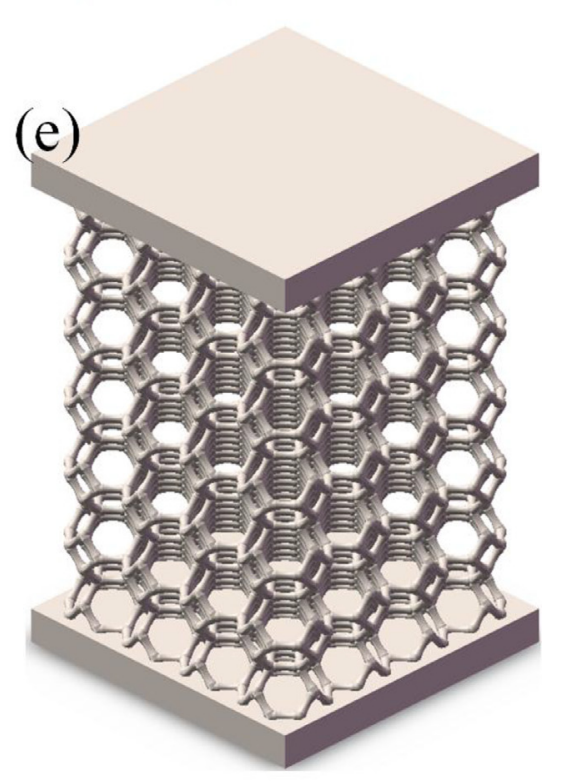

$4 \times 4 \times 6$

failure mechanisms [24]. Quantitative in situ multi-scale experimentswere employed for studying the damage process of metal-coated hybrid meso-lattice composites, and the damage process was simulated and elaborated based on the progressive damage theory and fracture mechanics [25]. Ultralight-weight sandwich structure with robust load bearing capacity and core buckling resistance was proposed by Wu et al. [26] , and three-dimensional failure mechanism maps for the pyramidalpyramidal hierarchical lattice material were developed for exploring its mechanical performances potentials. An increment equivalent continuum method was developed for studying the nonlinear mechanical properties of lattice truss composite materials [27]. Three beam-like lattice structures with triangular prism, square prism and hexagonal prism are designed and fabricated by SLM process using AlSi10Mg metal powder materials, and it is found that the stiffness-to-mass ratio of lattice structures with triangular prisms are better than other types of configurations, demonstrating advantageous structural efficiencyin terms of strength and stiffness [28]. Topology optimization techniques with SLM additive manufacturing constraints are proposed for designing three types of 3D lattice structures, and Gibson-Ashby model is employed for predict the performance of the three structures with different levels of porosity [29]. Sercombe et al. [30] studied the deformation process and failure mechanisms of topology-optimized lattice scaffolds structures via interrupted compression testing and X-ray micro-tomography (XMT) characterization. Making use of homogenized method, Messner et al. [31] developed parameterized model for generating optimal mesostructures. The mechanical properties of micro-lattice structures subjected to normal stresses in arbitrary directions are investigated using an analytical approach based on classical beam theory, the stiffness and plastic 
Table 1

Relative density of designed lattice structures.

\begin{tabular}{lllll}
\hline Relative density & Octet-truss & Tapered octet-truss & Truncated-octahedron & Tapered truncated-octahedron \\
\hline Analytical & $21.19 \%$ & $26.38 \%$ & $5.26 \%$ & $7.03 \%$ \\
CAD & $20.69 \%$ & $26.64 \%$ & $5.25 \%$ & $7.22 \%$ \\
Error & $2.42 \%$ & $0.98 \%$ & $0.19 \%$ & $2.63 \%$ \\
\hline
\end{tabular}
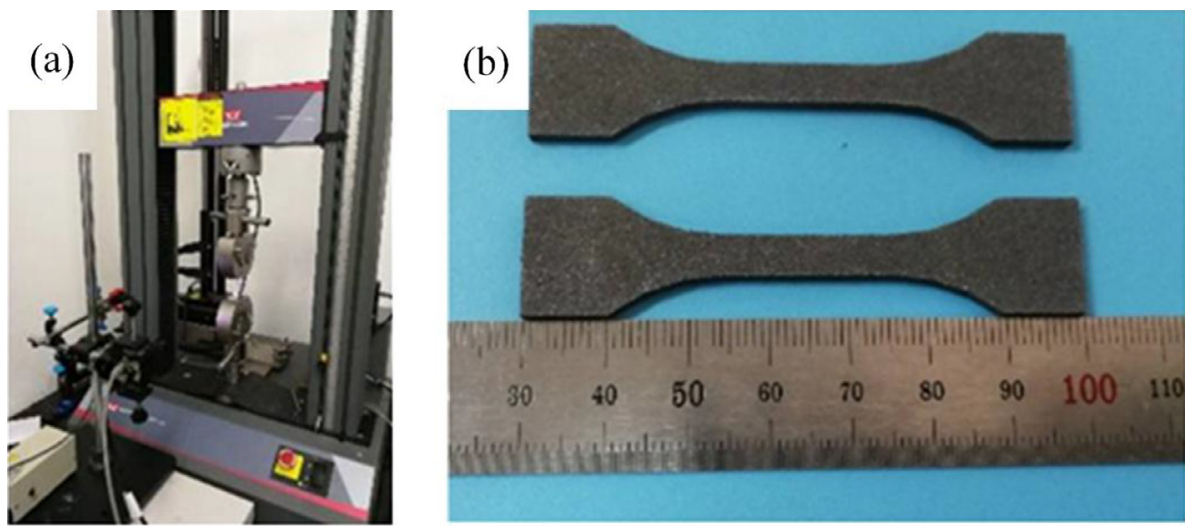

Fig. 3. Mechanical properties testing; (a) View of the setup for tensile test along; (b) printed tensile sample; (c) speckle on the tensile sample; (d) engineering stress-strain curves of the two samples.
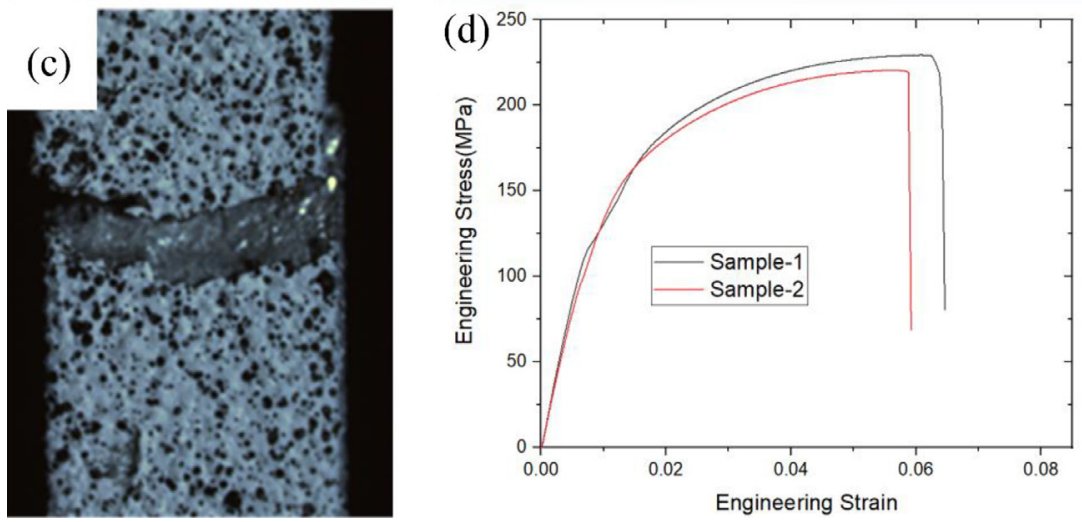

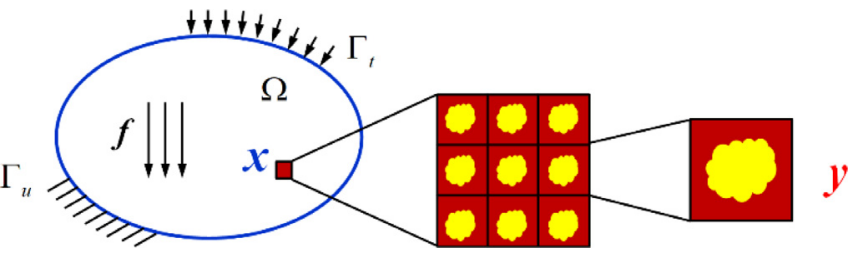

Fig. 4. Homogenization concept of a heterogeneous material.

collapse strength of the micro-lattice structures are compared with the corresponding properties of other lightweight structures [32]. The shearing modulus and strengths of lattice structures were studied in terms of relative density, and two strut failure modes are proposed for understanding the underlying failure mechanisms: elastic buckling of the struts governed the response for low relative density case and delamination failure controlled the strength for higher relative density [33]. Masta et al. [34] investigated the fracture toughness of a Ti-6Al-4 V alloy octet-lattice truss structure manufactured using a 'snap-fit' method, and the fracture resistance was determined using the J-integral compliance method applied to single-edge notched bend specimens.

Considering the coupling effect of axial force and bending moment, theoretical approach for predicting the yield surface of lattice structures under complex stress state is developed for two types of 3D novel lattices, namely BCCZ (body-centered cubic with a strut in Z direction) lattice, and 3D rhombic dodecahedron (RD) lattice [35]. The effect of vacuum thermal cycling on the compression and shear performance of the composite sandwich panels with pyramidal-truss cores was studied using theoretical and experimental methods [36]. Latture et al. [37] studied and compared the mechanical behaviors of two types of octet trusses with and without filleted nodes. Based on structural superposition design of elementary truss and plate structures, 3D elastically-isotropic lattice and plate metamaterials are developed [14,31,35-39]. Mechanical response of 3D lattice structures is anisotropic, where lattice beams are stretching-dominant or bending-dominant controlled depending on the loading conditions, new approach through adjusting the ratio of bending to stretching beam ratio is proposed for designing novel isotropic lattice [40]. Novel Hourglass truss lattice sandwich structure is fabricated with snap-fitmethod, it is found that the compressive strengths and peak compressive loads of as-fabricated Hourglass lattice sandwich structures are superior to pyramidal sandwich structures [41]. Tetrahedral lattice truss sandwich panels are manufactured by folding and air brazing of hexagonally perforated 6061 aluminum alloy sheets, and inelastic columnbuckling model is developed for predicting the lattice truss compressive peak strength over a range of relative densities [42]. Sandwich panels consisting of vertical strutintegrated body-centered cubic 3D lattices are fabricated with selective laser melting (SLM) using AlSi10Mg metal powder materials, and the effects of layer and cell numbers on 


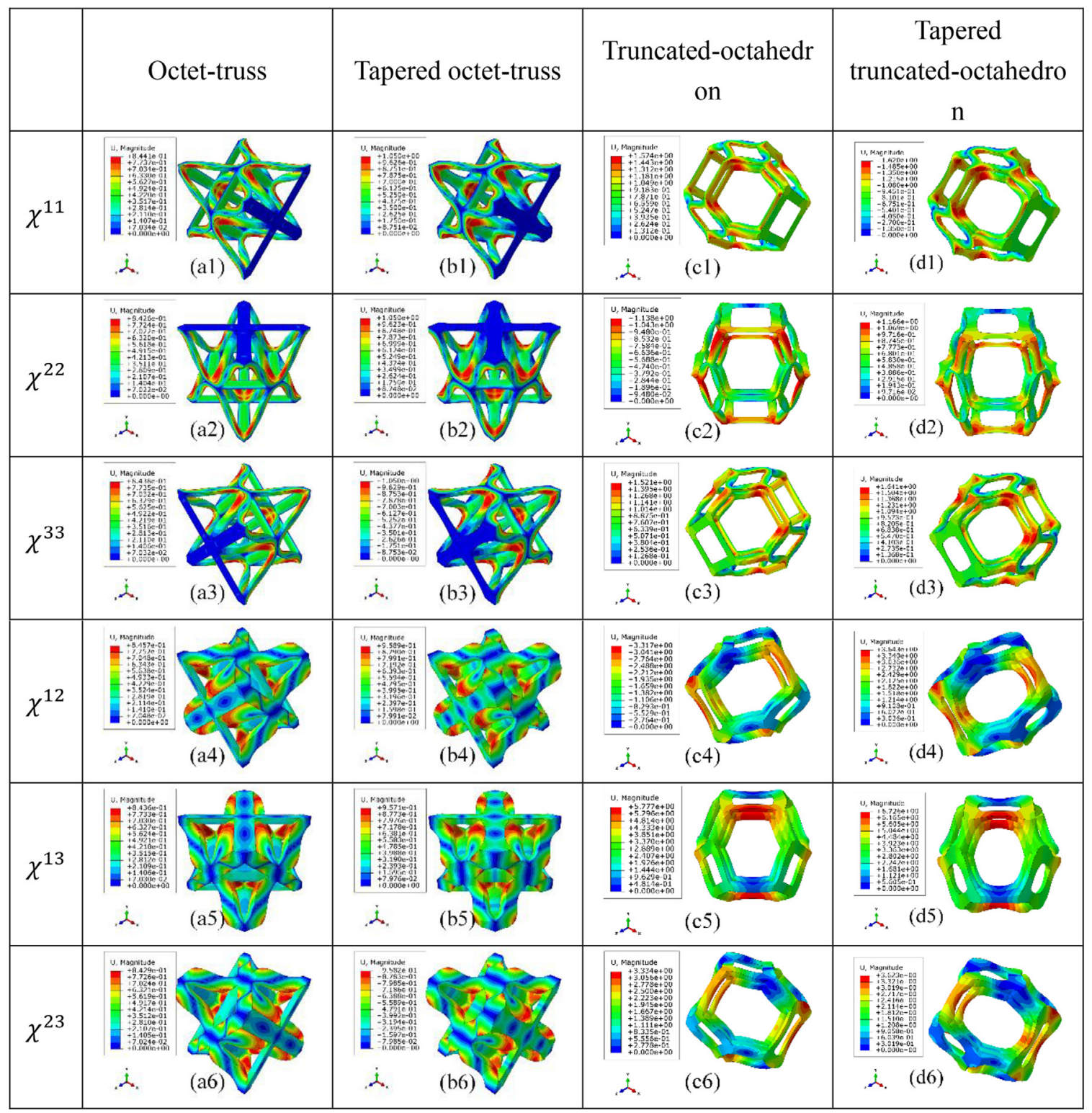

Fig. 5. $\chi^{m n}$ filedof the unit cell calculated through Eqs. (A.28); (a1-a6) are the displacement field of octet-truss lattice under surface traction $T_{m n}$; (b1-b6) are the displacement field of tapered octet-truss lattice under surface traction $T_{m n}$; (c1-c6) are the displacement field of truncated-octahedron lattice under surface traction $T_{m n}$; (d1-d6) are the displacement field of tapered truncated-octahedron lattice under surface traction $T_{m n}$.

the quasi-static compressive performances of BCCZ sandwich panels are investigated systematically [43]. Making use of buckling-dominated deformation induced large deformation, sandwich panels with lattice cores composed by curved struts is proposed for energy absorption, it is found that the energy-absorbing ability of the proposed lattice materials with curved struts is much better than common lattice materials composed by straight struts [44]. Beharic et al. [45] investigated and compared the impact energy absorption performances of three types of sandwich structures with different core lattice geometrical configurations. The elastic properties and plastic yielding collapse surfaces of the octet-truss lattice structured material are investigated through experimental and finite element simulation comparison [46]. Making use of in-situ microCT tomography and finite element analysis, Geng et al. [47] investigated the compression mechanical properties of SLM metal Al-Si10-Mg additive manufactured 3D re-entrant lattice, and found that manufacturing process induced surface roughness and manufacturing geome- try differences can influences the mechanical performances of lattice structures remarkably. Based on in-situ SEM fatigue experiments and micro-CT tomography characterizations of defects, Wang et al. [48] investigated the relation between fatigue performances and internal defects features of SLM metal Al-Si10-Mg additive manufactured tensile sample.

It can be concluded from above-mentioned literatures that the beam geometry of unit cellcan significantly affect the overall strength, stiffness, anisotropy, deformation and failure mechanism of lattice structure. In this paper, mechanical properties and deformation mechanisms of SLM additive manufactured stretching-dominated octet-truss and bending-dominated truncated-octahedron lattice structures consisting of ordinary uniform beams and tapered beams are investigated. Firstly, analytical relative density formulas of the lattice structures with ordinary homogenous beam and tapered beam are derived; Afterwards, asymptotic homogenization(AH) theory is employed for 


\begin{tabular}{|c|c|c|c|c|}
\hline & Octet-truss & Tapered octet-truss & $\begin{array}{c}\text { Truncated-octahedr } \\
\text { on }\end{array}$ & $\begin{array}{c}\text { Tapered } \\
\text { truncated-octahedro } \\
\mathrm{n}\end{array}$ \\
\hline$\frac{\partial \chi^{11}}{\partial y}$ & 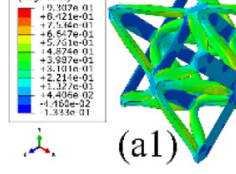 & $\begin{array}{ll} & \\
& \\
& \end{array}$ & 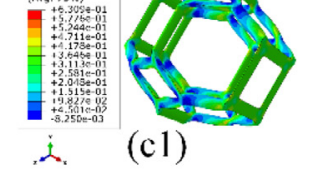 & 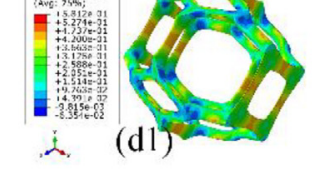 \\
\hline$\frac{\partial \chi^{22}}{\partial y}$ & 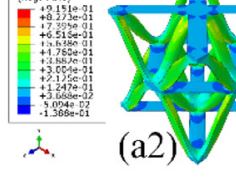 & 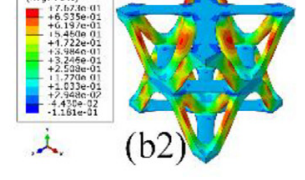 & 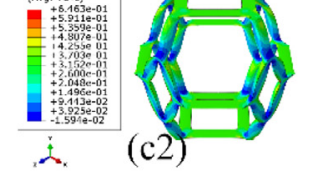 & 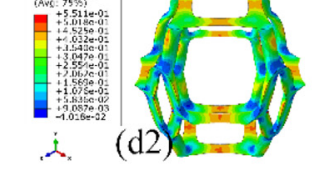 \\
\hline$\frac{\partial \chi^{33}}{\partial y}$ & 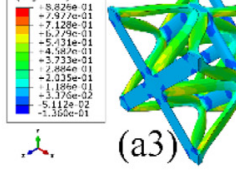 & 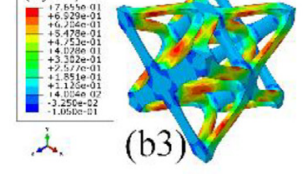 & 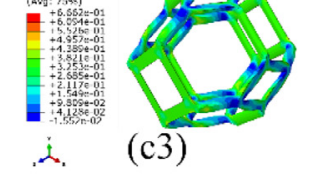 & 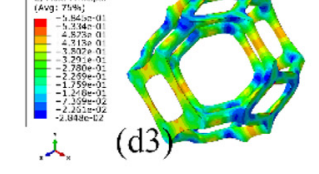 \\
\hline$\frac{\partial \chi^{12}}{\partial y}$ & 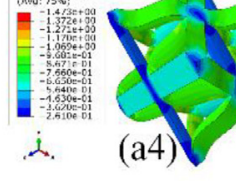 & 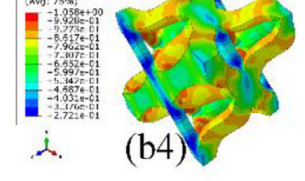 & 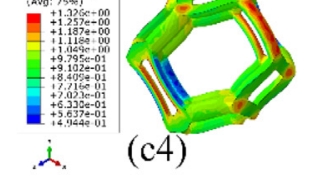 & 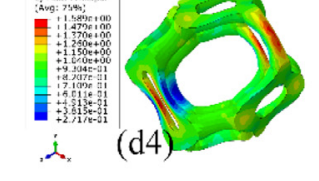 \\
\hline$\frac{\partial \chi^{13}}{\partial y}$ & 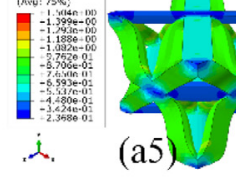 & 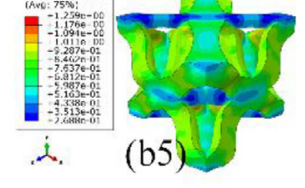 & 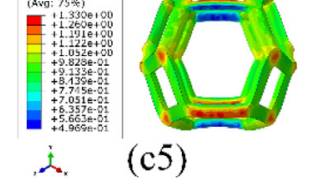 & 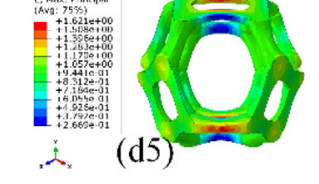 \\
\hline$\frac{\partial \chi^{23}}{\partial y}$ & 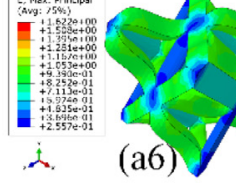 & 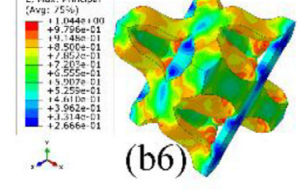 & 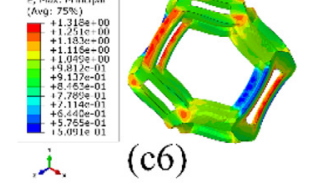 & 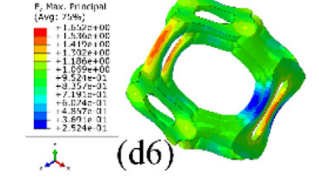 \\
\hline
\end{tabular}

Fig. 6. $\frac{\partial \chi^{m n}}{\partial y}$ fieldof the unit cell obtained by the gradient calculation of the $\chi^{m n}$ field; (a1-a6) are the strain field of octet-truss lattice under surface traction $T_{m n}$; (b1-b6) are the strain field of tapered octet-truss lattice under surface traction $T_{m n}$; (c1-c6) are the strain field of truncated-octahedron lattice under surface traction $T_{m n}$; (d1-d6) are the strain field of tapered truncated-octahedron lattice under surface traction $T_{m n}$.

investigating the anisotropy mechanical properties of lattice structures; Finally, comparisons between experiments, theoretical analysis and finite element simulations are performed for exploring the effects of tapered beams on lattice structures mechanical properties improvements. Through node enhancement design, the proposed lattice structures show robust industrial application potentialsin lightweight sandwich structures in aerospace industry, impact energy absorption lightweight structures in automotive industry, transportation industries. Moreover, these proposed lattice structures can also be employed for biomedical applications, such as: bone implant porous lattice structures, etc.

\section{Mechanical design of compression lattice structures}

In this paper, stretching-dominated octet-truss lattice unit cell with uniform beam cross-section and tapered beam are designed, and tapered beam with node strengthening geometrical layout are employed for el- evating the superior compression resistance mechanical properties of ordinary octet-truss lattice unit cell. Whereas the stanching-dominated octet-truss has been investigated extensively, the bending-dominated truncated octahedron catches far less attention. Both the lattice structures are selected to give a comparison of the difference deformation mechanism and the node strengthening effect, which displays via quasistatic compression analysis.

Figs. 1 and 2 shows the topology of the unit cell and prismatic sample under investigation. From the unit cell topology, both the octet cell and truncated-octahedron cell are cubic symmetry. First, in Fig. 1, the regular octet unit cell is composed by an octahedron and eight tetrahedrons. The eight tetrahedrons are attached to the eight faces of the octahedron. Second, in Fig. 2, the truncated-octahedron is an Archimedean solid. It is constructed from a regular octahedron by the removal of six right square pyramids from each point. It has 14 faces (8 regular hexagonals and 6 squares), 36 edges and 24 vertices. 


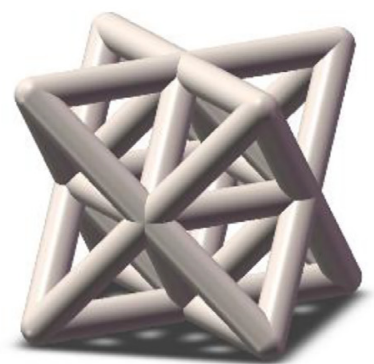

(a)
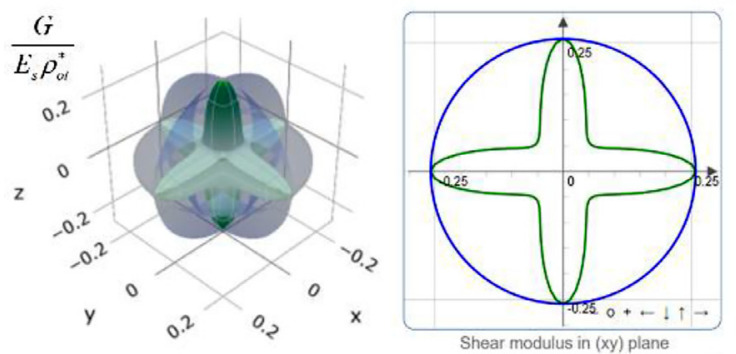

(b)

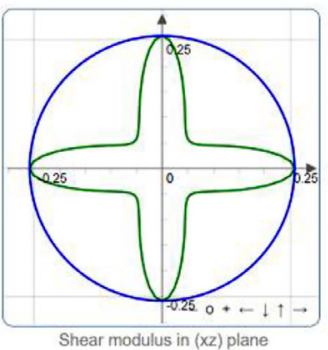

Shear modulus in (xz) plane

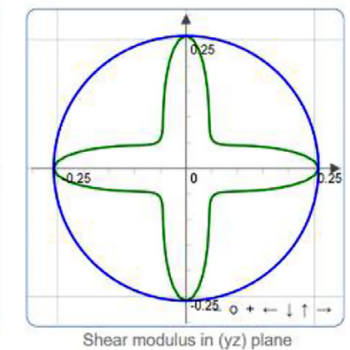

Shear modulus in (yz) plane
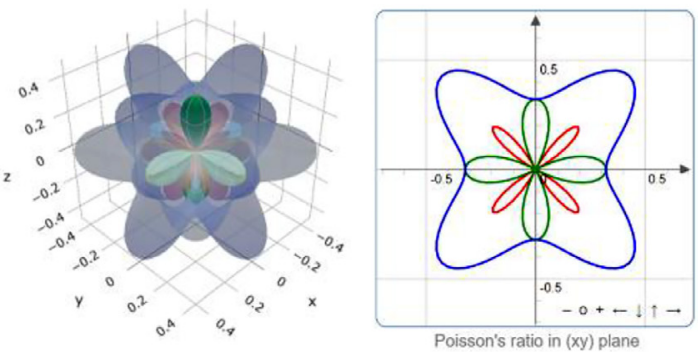

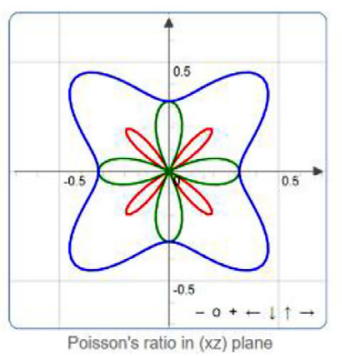

Poisson's ratio in (xz) plane

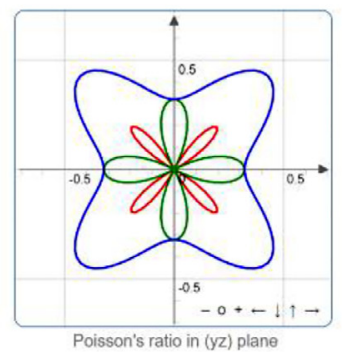

Poisson's ratio in (yz) plane

(c)

Fig. 7. Anisotropy of the octet-truss lattice structure. (a) unit cell and Young's modulus surface. (b) shear modulus surface, xy plane, xz plane and yz plane. (c) Poisson ratio surface, xy plane, $x z$ plane and yz plane. The transparent blue surface represents the maximum value, the green and red surfaces represent the minimum value and the red surface represents the negative value. The same expression was adopted in Figs. 8-10.

For the unit cell in Figs. 1 and 2, the beam length is denoted as $L$, the edge length of the unit cell are $L_{o t}=\sqrt{L}$ and $L_{t o}=2 \sqrt{2} L$ respectively. For there are 24 complete beams in unit cell, the relative density of the metamaterials can be defined by the ration of the volume of the beams and the volume of the unit cell,

$\rho_{o t}^{*}=\frac{24 \mathrm{~V}}{L_{o t}{ }^{3}}$ and $\rho_{t o}^{*}=\frac{24 \mathrm{~V}}{L_{t o}{ }^{3}}$

with $V$ denoting the volume of a single beam. For the unit cell with uniform beam cross section of radius $R$, the relative density can be given

$$
\begin{aligned}
\rho_{o t}^{*}= & 6 \sqrt{2} \pi\left(\frac{R}{L}\right)^{2}-\frac{32}{2-\sqrt{2}}\left(\frac{R}{L}\right)^{3} \\
\rho_{t o}^{*}= & \frac{3}{4} \sqrt{2} \pi\left(\frac{R}{L}\right)^{2}-\frac{100}{27}\left(\frac{R}{L}\right)^{3} \\
& +\frac{\sqrt{2}}{2}\left(\frac{R}{L}\right)^{3}\left[2 \theta_{1}+\theta_{2}+\sin 2 \theta_{1}+\frac{1}{2} \sin 2 \theta_{2}-\pi\right],
\end{aligned}
$$

where $\rho_{o t}^{*}$ and $\rho_{t o}^{*}$ are the relative density of the octet-truss and truncatedoctahedron structures, respectively. The derivation of the formulation is presented in Appendix B1 and B2.
Apart from the uniform beam cross-section, we also consider the tapered beam with enlarged radius at ends of the constituent beams. As shown in Figs. 1 and 2, the radius of the beam section is a quadratic function of the position along beam mid-axis. Then the analytical expression of the relative density can be modified as

$$
\begin{aligned}
\rho_{t o t}^{*}= & 6 \sqrt{2} \pi\left(\frac{R}{L}\right)^{2} f+\pi\left(\frac{R}{L}\right)^{3}(6 \sqrt{2}+12)(1-f)-\frac{32}{2-\sqrt{2}}\left(\frac{R}{L}\right)^{3} \\
\rho_{t t o}^{*}= & \frac{3}{4} \sqrt{2} \pi\left(\frac{R}{L}\right)^{2} f-\frac{100}{27}\left(\frac{R}{L}\right)^{3} \\
& +\frac{\sqrt{2}}{2}\left(\frac{R}{L}\right)^{3}\left(2 \theta_{1}+\theta_{2}+\sin 2 \theta_{1}+\frac{1}{2} \sin 2 \theta_{2}-\pi f\right)
\end{aligned}
$$

where $\rho_{\text {tot }}^{*}$ and $\rho_{\text {tto }}^{*}$ are the relative density of the tapered octet-truss and tapered truncated-octahedron structures, respectively. The relative density of designed lattice structures is shown in Table 1.

\section{Results and discussion}

\subsection{Mechanical properties testing}

These lattice specimens were manufactured via SLM metal additive manufacturing technology. The Al-Si10-Mg powder materials used for 


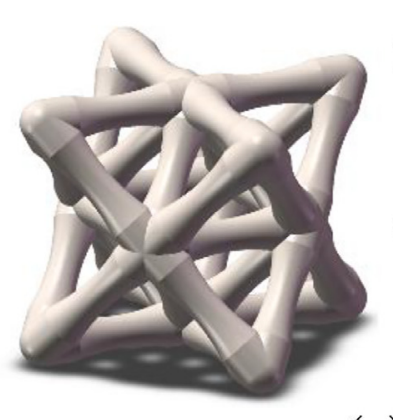

(a)

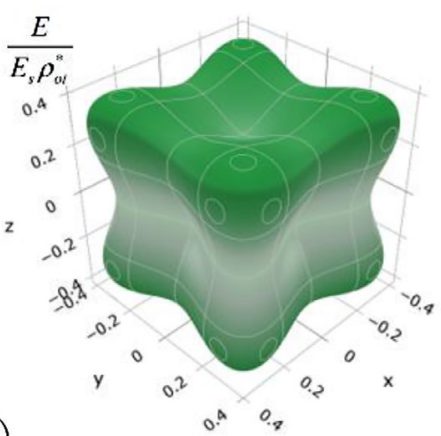

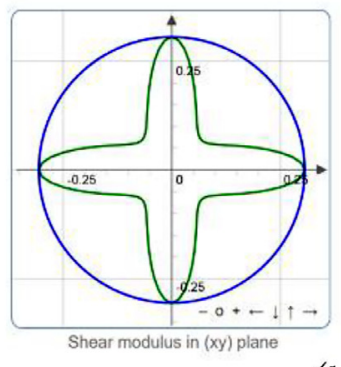

(b)

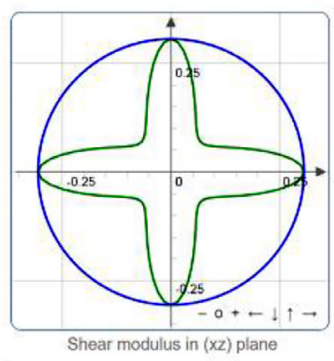

Shear modulus in $(x z)$ plane

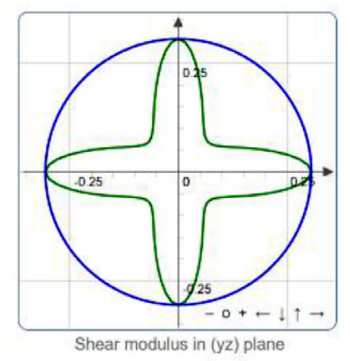

Shear modulus in (yz) plane

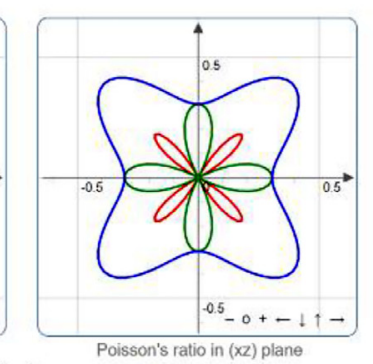

(c)

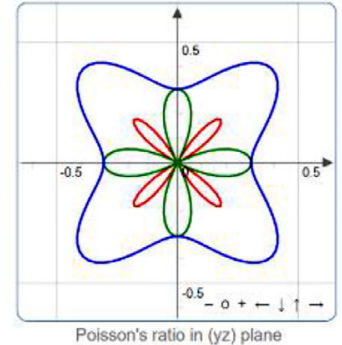

Poisson's ratio in (yz) plane
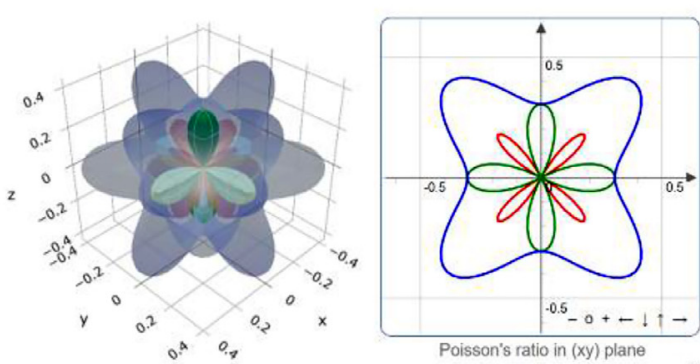

Poisson's ratio in $(x z)$ plane

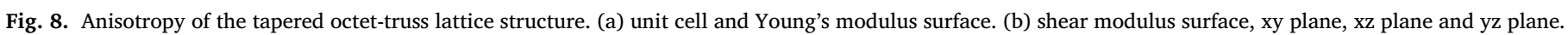
(c) Poisson ratio surface, xy plane, $x z$ plane and yz plane.

SLM printing with EOS M M290 (constructed by EOS GmbH, Germany) machine, a selective laser melting system from SLM Solutions GmbH. The Al-Si10-Mg fine powder was provided by EOS GmbH, with powder particle sizes ranging from 20 to $65 \mu \mathrm{m}$. The SLM laser power is set as $370 \mathrm{~W}$, and the scanning speed is set as $1300 \mathrm{~mm} / \mathrm{s}$, with layer thickness of $30 \mu \mathrm{m}$ in an argon gas atmosphere. The designed samples are supported on a plate heated to $300{ }^{\circ} \mathrm{C}$. To relieve residual stresses generated by the fabrication process, thermal stress relieving was performed on these specimens at $300{ }^{\circ} \mathrm{C}$ for $2 \mathrm{~h}$, prior to removing lattice structure specimens from the support structures. Tensile test was performed on the Instron2367, as shown in Fig. 3. The engineering strain was evaluated through digital image correlation method. Finally, we obtain the engineering stress-strain curves of the SLM printed samples.

\subsection{Elastic mechanical property with the proposed AH method}

The homogenization theory has been developed since 1970s mathematically. This approach can be used to analyze the matter system with two or more scales. It can relate the meso-scale containing the second phase space to the macroscopic scale. Fig. 4 depicts the homogenization procedure schematically. A composite elastomer $\Omega$ with periodic structure subjected to the surface traction $t$ at the traction boundary $\Gamma_{t}$, the displacement $d$ at the displacement boundary $\Gamma_{d}$ and a body force $f$. The microstructure at the point $x$ on the macroscopic can be treated as periodic repetition of the heterogeneous unit cell in spatial. Here, $\mathrm{AH}$ is adopted to analyze the effective mechanical properties of the proposed metastructure.It has proven to be a powerful technique to investigate the effective mechanical properties of the heterogeneous materials and metastructures with periodic structure arrangement [18,49-53].

Using the surface traction method depicted in previous section, we evaluate the detailed values of $\chi_{k}^{m n}$ for different lattice structure respectively, as shown in Fig. 5. The numerical model is discretized by C3D4 element and the element size is about $0.2 \mathrm{~mm}$.In the present research, there are about 40,000 elements in pure unit cell and we have examined the reliability of the element. According to Eqs. (A.28), $\chi_{k}^{m n}$ is represented by the displacement filed in the $k$ direciton under the surface traction in the $e_{m n}$ direction. And $\chi^{m n}$ is represented by the resultant displacement field. By introducing a taper,the deformation tends to concentrated in the middle of the beam, as shown in Figs. 5 and 6 . Also it can be clearly seen that beams distributions in special directions have significant influence on the overall deformation respond of the unit cell.

Different from the continuum materials, lattice structures are usually strong anisotropic due to the special arrangement of the beams. Base on the homogenization process, we evaluate the effective elastic tensor of 

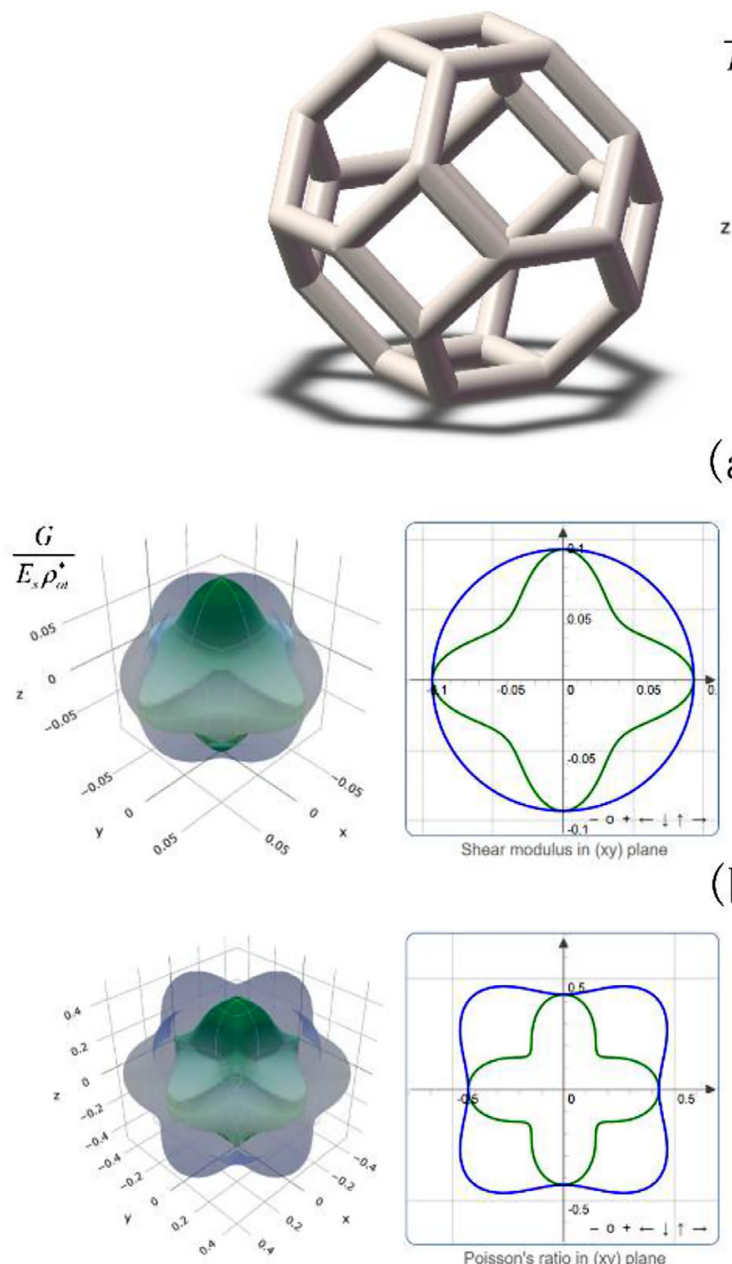

(c) (a)

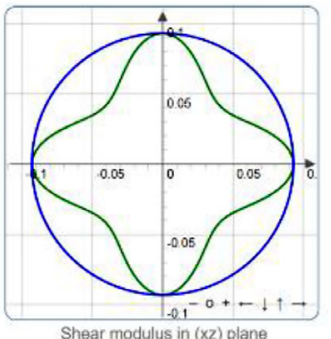

(b)
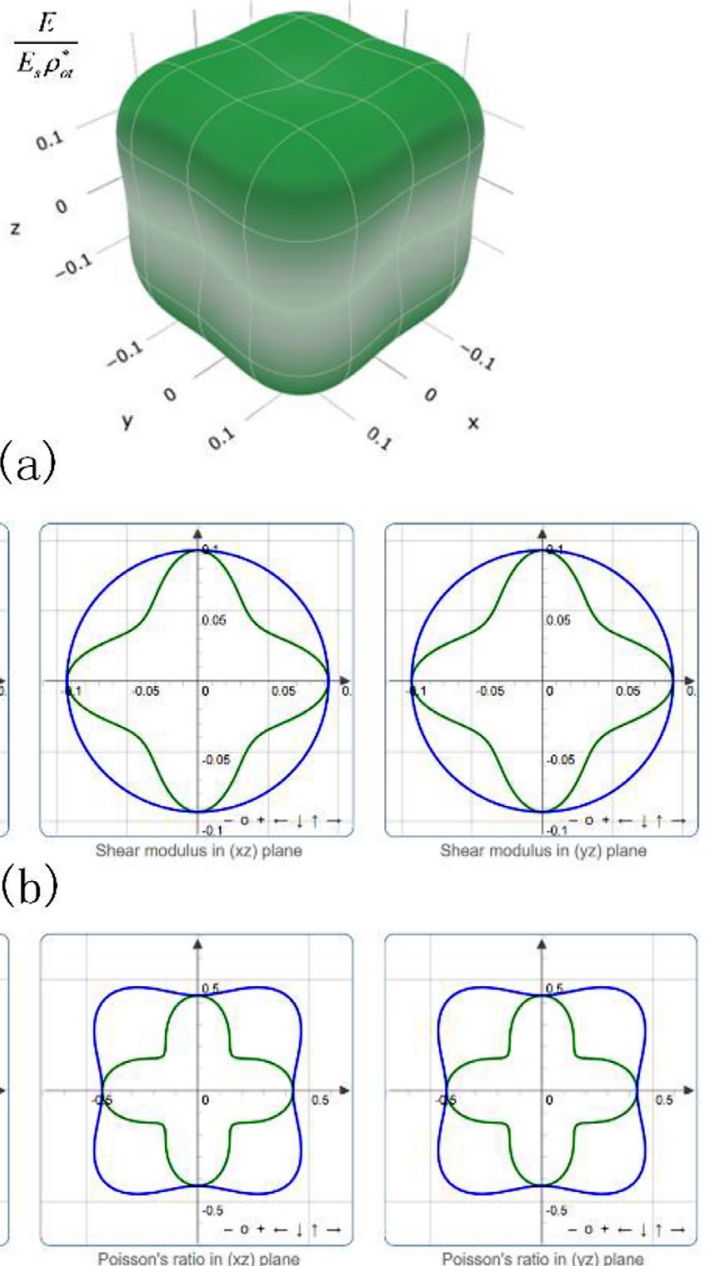

Shear modulus in (yz) plane

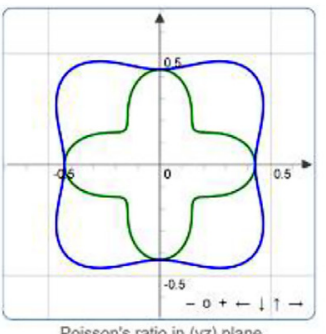

Poisson's ratio in (yz) plane

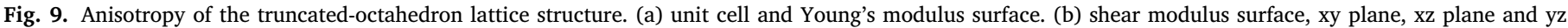
plane. (c) Poisson ratio surface, xy plane, $x z$ plane and yz plane.

the proposed unit cells. For cubic symmetry materials, the theoretical bulk modulus reads

$K=\frac{1}{3}\left(D_{1111}+2 D_{1122}\right)$

Furthermore, the Young's modulus for the [100] direction can be expressed analytically as

$E=\frac{\left(D_{1111}-D_{1122}\right)\left(D_{1111}+2 D_{1122}\right)}{D_{1111}+D_{1122}}$

And the shear modulus reads

$G=D_{1212}$

Using the open-source online application provided by Gaillac [54] ,we further compute the anisotropic elastic tensors and represent them graphically. As shown in Figs. 7-10, the Poison ratio, generalized Young's modulus and shear modulus are plotted in an orthogonal coordinate system. The anisotropy of elastic properties can be defined as the ratio of maximum value to the minimum value.Clearly, the anisotropy in special directions can be demonstrated and is highly dependent on the special distribution of beams. For example, in octettruss structure, the ratio of Young's modulus in [111] direction to mod- ulus in [100] is about 2.67, as shown in Fig. 7(a).That is mainly because the much higher stiffness of the tetrahedrons attached to the eight faces of the octahedron. Furthermore, illustrated in Fig. 7(b),the anisotropy of the shear modulus is much higher than that of Young's modulus. The anisotropy of shear modulus is about 3.43. Fig. 7(c) shows the strong anisotropic distribution of the Poison ratio. The maximum Poison ratio is about 0.61 and the minimum value is about -0.28 .

However, Young's modulus surface of truncated-octahedron lattice structure seemly to be cube, as shown in Fig 8(a). The surface has maximum value in diagonal directions. The anisotropy of Young's modulus and shear modulus are 1.56 and 1.60, respectively. Different from that in octet-truss lattice structure, the minimum Poison ration is about 0.22, which is positive. From the comparison of Figs. 7(c) and 9(c), the anisotropy of octet-truss is much stronger than that of the truncatedoctahedron lattice structure.

Inspired by the research of Thomas [38], the tapering design strategy is proposed to control the anisotropy of the lattice structure. Noticing the elastic properties in Figs. 8(a-b) and 10(a-b), it has to be point out that the strategy works well in modulating elastic properties. For example, the anisotropy of Young's modulus and shear modulus of tapered octet-truss structure are 2.47 and 3.16, respectively, which are lower than that of octet-truss structure. However, for the comparison 

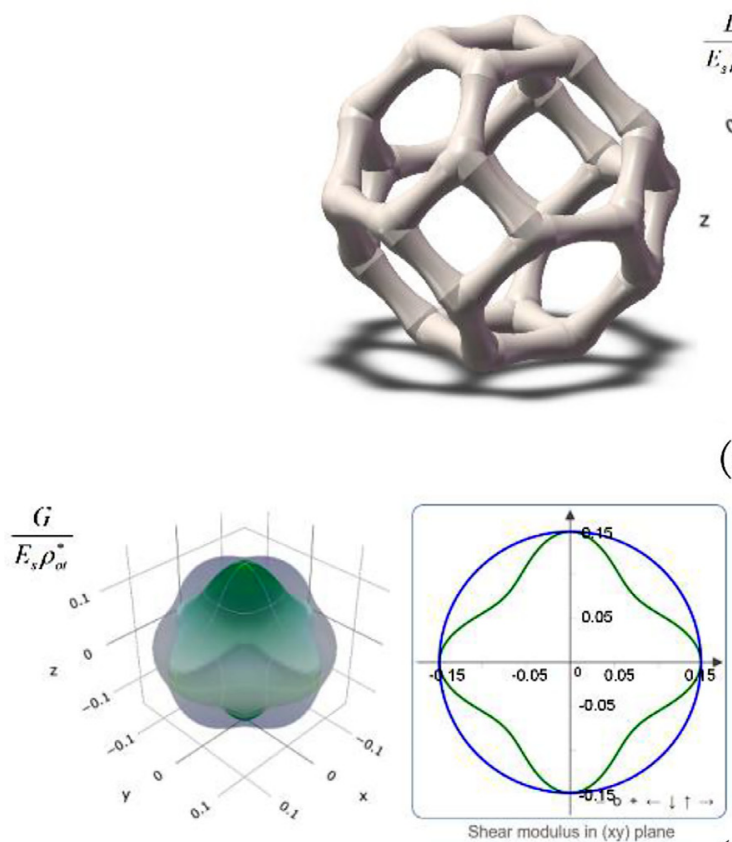

(b)
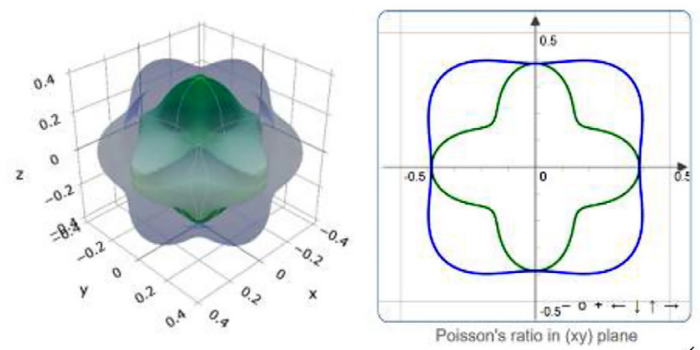

(c) (a)
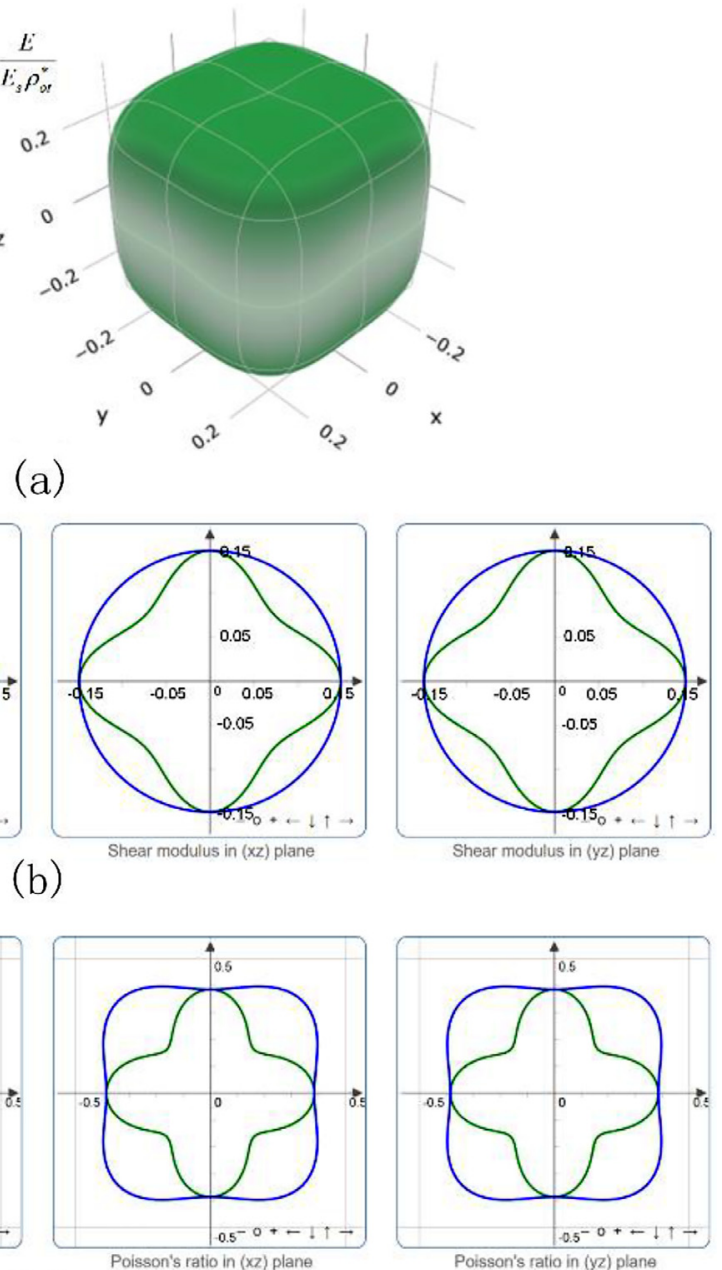

Shear modulus in (yz) plane

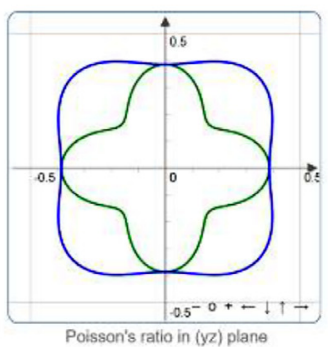

Poisson's ratio in (yz) plane

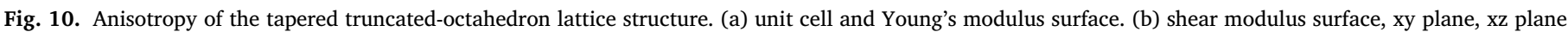
and yz plane. (c) Poisson ratio surface, xy plane, xz plane and yz plane.

of maximum Young's modulus and shear modulus, the tapering strategy increases the response by more than $120 \%$ and $160 \%$ for octettruss and truncated-octahedron structures, respectively. With regards to the Young's modulus and shear modulus, the effect of tapering strategy is less beneficial for octet-truss structure. Considering the stretchingdominated deformation in octet-truss structure and bending-dominated deformation in truncated-octahedron structure, the tapering strategy provides more privilege to control the elasticity in periodic structure of bending-dominated. From Figs. 8(c) and 10(c), it can be concluded that tapering strategy can significantly decrease the Poison ratio distribution range in special directions.

\subsection{Large deformation response for static in situ compression test}

Figs. 11-13 illustrates the in situ monotonic compression of octettruss and truncated-octahedron lattice with uniform beam cross-section and node enhancement respectively. The elastic modulus along [100] direction is evaluated and the comparison between experiment and analytical result from Eqs. (A.7) are presented in Table 2.

\subsubsection{In situ compression test on octet-truss and tapered octet-truss structures}

According to Fig. 11(a), four deformation stages can be seen in the stress strain curve, namely elastic stage, nonlinear stage, damage stage and densification stage. Specifically, linearity of the stress-strain occurs in the initial stage, then nonlinearity occurs with the increasing compression load. From the Fig. 11(d-e), the nonlinearity is mainly attributed to the bending deformation of the beams in the metastructure. A shear band along $45^{\circ}$ with respect to the loading direction occurs, which is caused by the fracture at the intersection nodes of the beams, leading to the abrupt decrease in stress-strain curve. Theoretically, this direction is coincident with the maximum shear stress surface. Subsequently, another shear band along the other side occurs repeatedly. Then densification is formed with the increasing compression load. To be more specific, the SEM characterization of the fracture surface is conducted to investigate the local imperfection around intersection nodes of the beams, as shown in Fig. 11(b). The red labels in the Fig. 11(b) demonstrate that there are many voids around intersection nodes. This is possible caused by the immature manufacturing technique. In addition, shown in Fig. 11(c), the side views of the samples show that the shear band governed by shear stress is symmetry, which is different from that reported by Song [25].

Considering the shear-stress dominated the deformation mechanism and the imperfection around intersection nodes, node enhancement is introduced to the metastructure. Subsequently, the tapered octet-truss structure is compressed and the corresponding results are given in the Fig. 12. Compared with the result of octet-truss structure, Fig. 12(c) shows extremely different deformation mechanism. The results show 

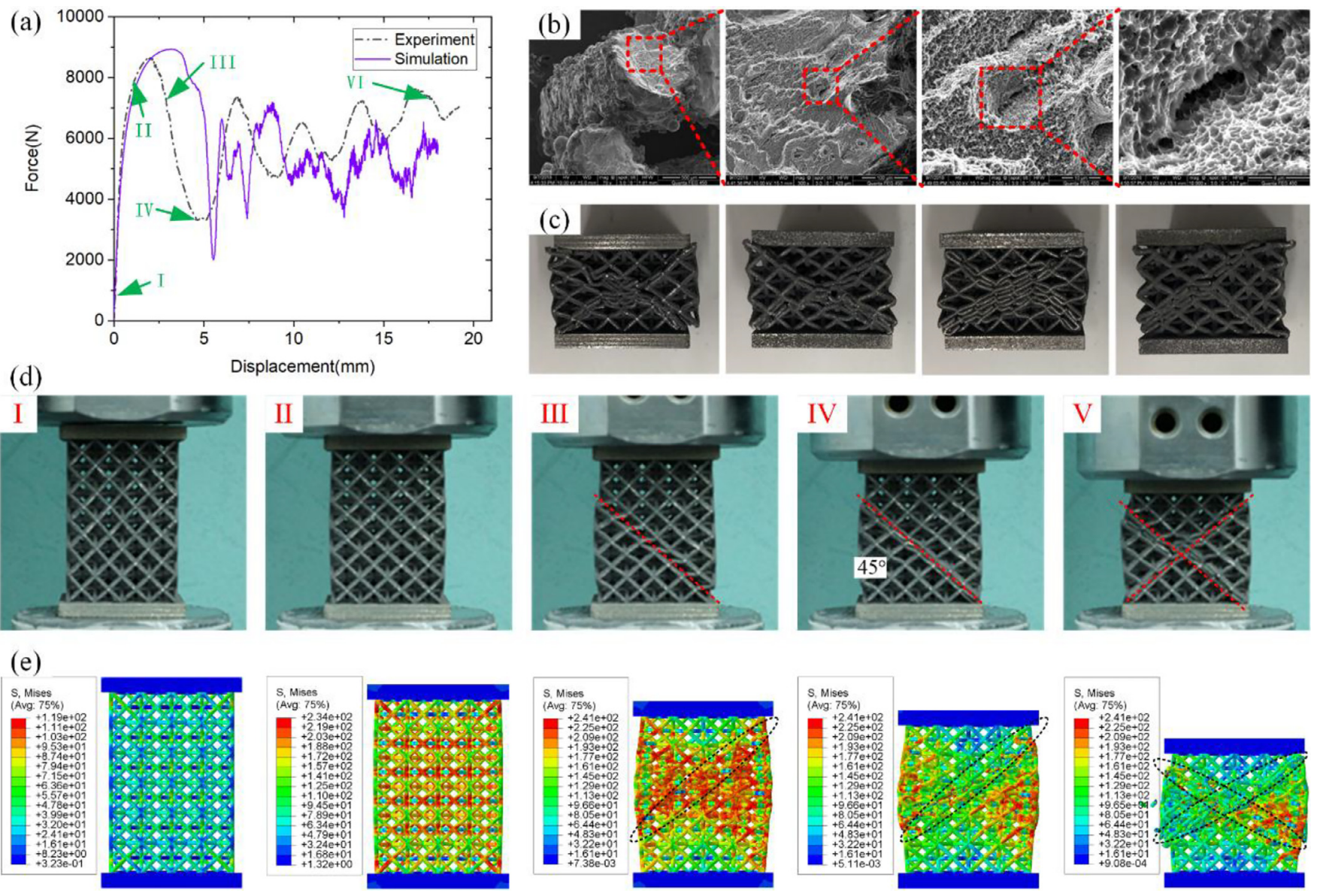

Fig. 11. In-situ compression of octet-truss 3D lattice with uniform lattice beam geometries. (a) Typical force-displacement curve. (b) SEM photographs of the fracture morphologies. (c) Macroscopic fracture morphologies of the sample after experiment. (d) Deformation history of the octet-truss lattice structure. The snapshots of I-VI correspond to the loading states labeled in (a). (e) Deformation history of the numerical simulation.

Table 2

Elastic modulus along [100] direction.

\begin{tabular}{lllll}
\hline & Octet-truss & Tapered octet-truss & Truncated-octahedron & Tapered truncated-octahedron \\
\hline Expermental & $925 \mathrm{MPa}$ & $1337 \mathrm{MPa}$ & $106 \mathrm{MPa}$ & $276 \mathrm{MPa}$ \\
Analytical & $976 \mathrm{MPa}$ & $1543 \mathrm{MPa}$ & $202 \mathrm{MPa}$ & $474 \mathrm{MPa}$ \\
Numerical & $952 \mathrm{MPa}$ & $1494 \mathrm{MPa}$ & $193 \mathrm{MPa}$ & $452 \mathrm{MPa}$ \\
\hline
\end{tabular}

that the node enhancement has a significant effect on the mechanical properties of the present structure. First, there is not shear band during the compression progress and progressively collapse deformation mechanism dominate the compression progress. To be more specific, shown in Fig. 12(c), the fracture mainly occurs at the middle of the beams. Moreover, Fig. 12(c) demonstrated that the deformation is not symmetry. Finally, the SEM characterization of the fracture surface illustrated there also manufacture imperfections, as shown in Fig. 12(b).

\subsubsection{In situ compression test on truncated-octahedron and tapered} truncated-octahedron structures

Compared to octet-truss and tapered octet-truss, the truncatedoctahedron and tapered truncated-octahedron are compressed experimentally. Results in Fig. 13 shows that the truncated-octahedron lattice is really a low load-bearing structure compared to the octet- truss lattice structure. Additionally, shown in Fig. 14, the fracture morphology of the truncated-octahedron lattice structure turns out to be progressively collapse deformation and the tapered truncatedoctahedron lattice was shear band dominated. The result is completely inversed compared to the octet-truss and tapered octet-truss lattice structure. The same fracture morphology can be seen in numerical results.

\section{Conclusion}

In this paper, mechanical properties and deformation mechanisms of SLM additive manufactured stretching-dominated octet-truss and bending-dominatedtruncated-octahedron lattice structures consisting of ordinary uniform beams and tapered beams are investigated.

Firstly, analytical relative density formulas of the lattice structures with ordinary homogenous beam and tapered beam are derived. 

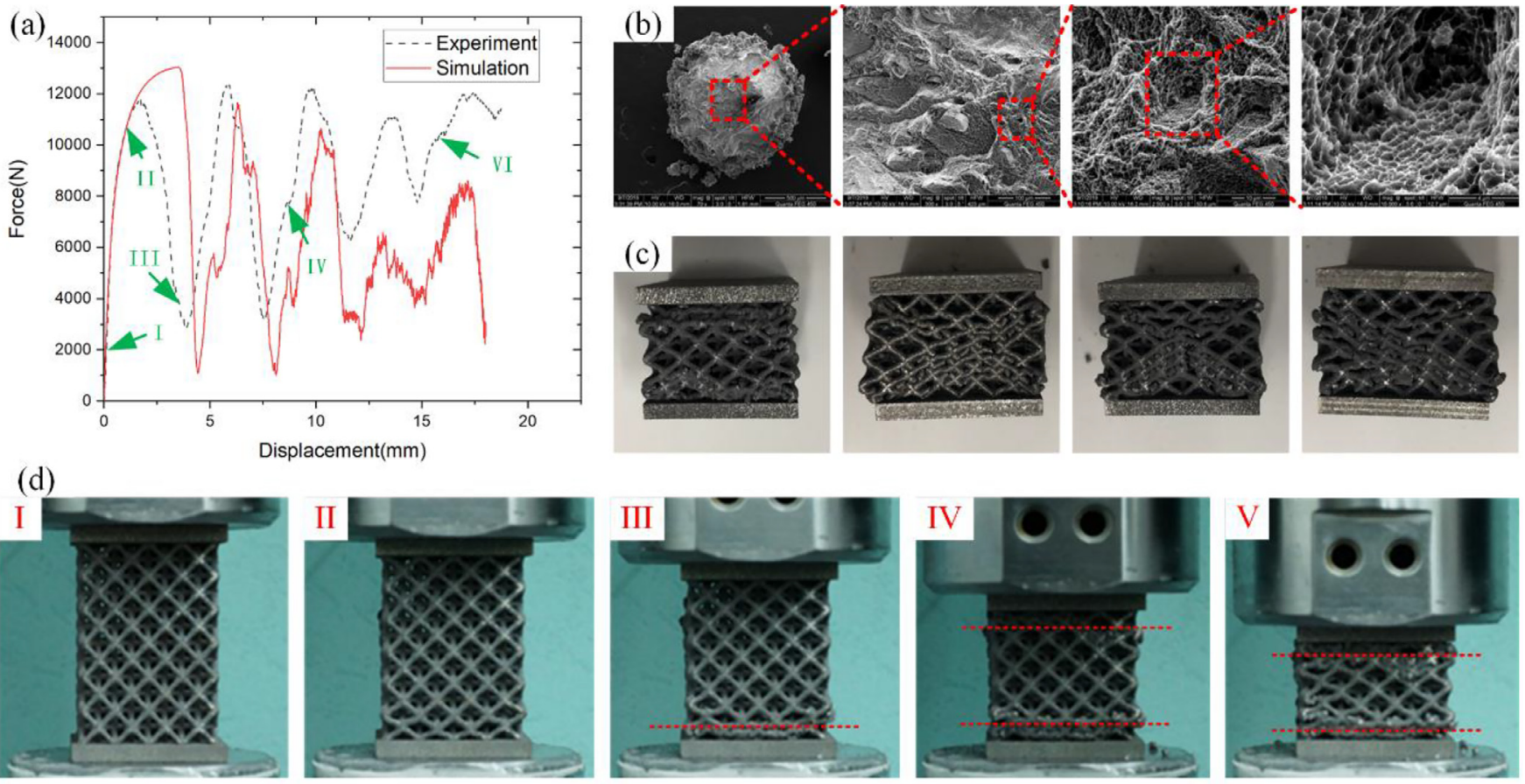

(e)
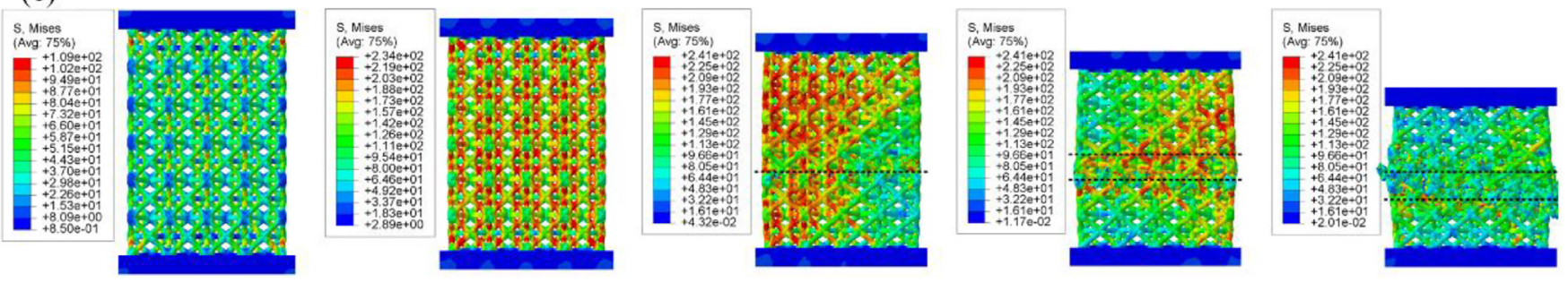

Fig. 12. In-situ compression of octet-truss 3D lattice with enforced node geometries. (a) Typical force-displacement curve. (b) SEM photographs of the fracture morphologies. (c) Macroscopic fracture morphologies of the sample after experiment. (d) Deformation history of the octet-truss lattice structure. The snapshots of I-VI correspond to the loading states labeled in (a). (e) Deformation history of the numerical simulation.

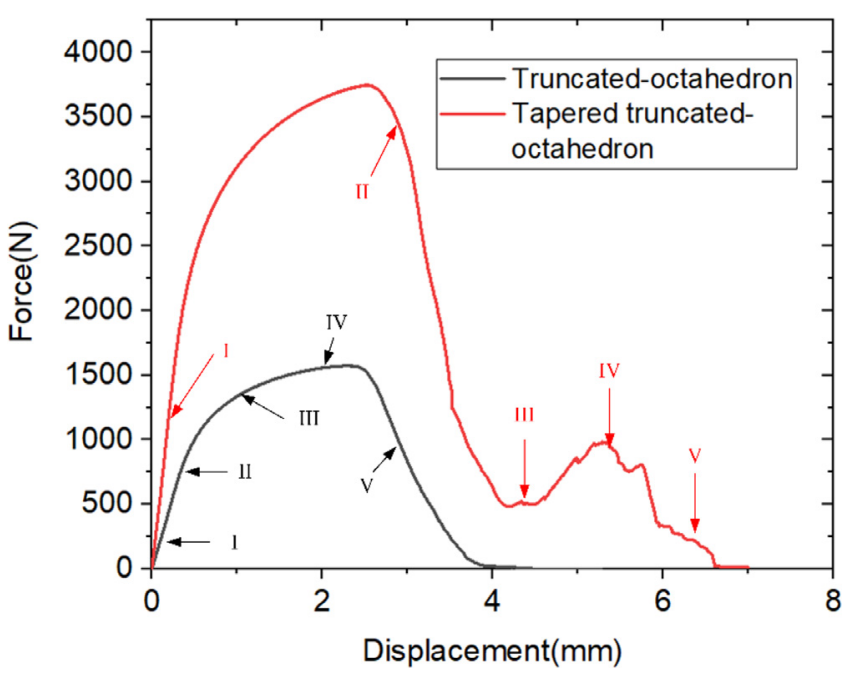

Fig. 13. In-situ compression force-displacement curves of truncatedoctahedronand tapered truncated-octahedron lattice structure.
Compared to the CAD model, the error of the analytical relative density function is less than $3 \%$.

Secondly, analytical expression of the AH theory was employed for investigating the anisotropic mechanical properties of the proposed lattice structures. It is found that elastic anisotropy is closely related to the spatial arrangement of lattice beams for both lattice structures with ordinary homogenous beam and tapered beam. However, thetapered beam design can significantly improve the elastic modulus and reduce the elastic anisotropy of the lattice structure.

Thirdly, comparisons between experiments, theoretical analysis and finite element simulations are performed. The elastic properties, deformation behaviors and failure mechanism of proposed lattice structuresare systematically investigated. In situ compressions shown that lattice structure arrangement has significant effect on the large deformation of the compression sample. Stretching-dominated octet-truss lattice exhibitsshear band fracture morphology. The macroscopic fracture morphology of tapered octet-truss is dominated by progressively collapse deformation. However, for truncated-octahedron and tapered truncatedoctahedron lattice structure, the macroscopic fracture morphology was completely inversed. 

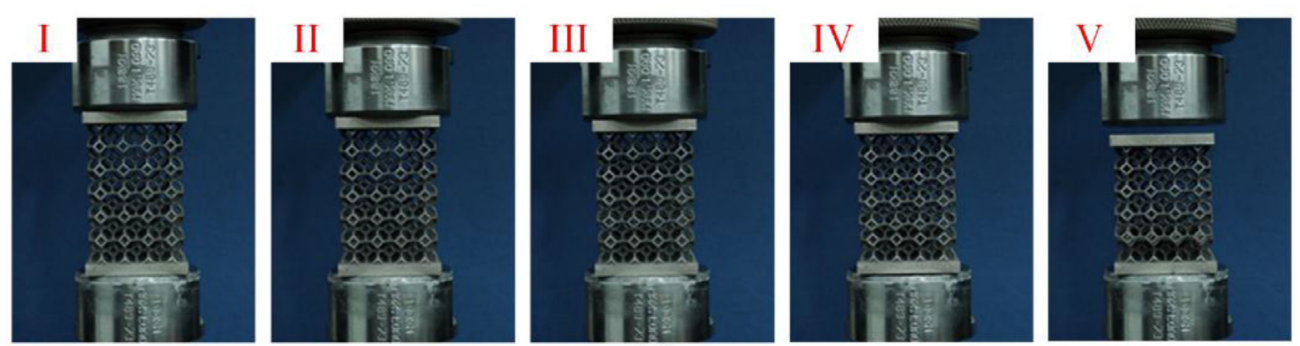

(a)
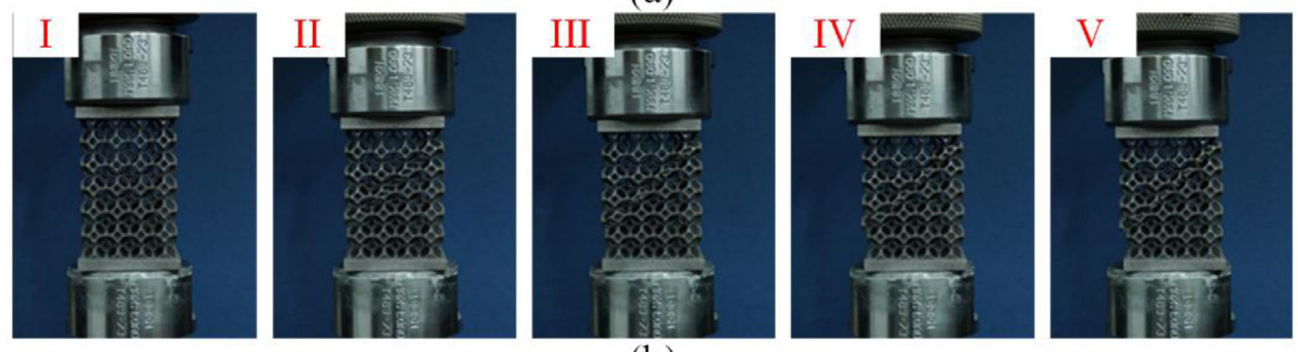

Fig. 14. (a) In-situ compression of truncated-octahedronand (b) tapered truncated-octahedron lattice structure.

\section{Acknowledgments}

This research is supported by the National Natural Science Foundation of China (grant nos. 11702023, 11632010 and 11972081), sponsored by the State Key Laboratory of Automotive Safety and Energy under project no. KF1808 \& no. KF1810.

\section{Appendix A}

Explicitly, assumptions are made in the AH theory. It is assumed that every field quantity depends on two spatial scales due to the existence of the microstructure. On the macroscopic level $x$, the field quantities, such as displacement, stress and strain, vary smoothly. On the microscopic level $y$, the field quantities are periodic. Base on AH theory, the relevant field quantities can be approximated by an asymptotic expansion:

$u_{i}^{\eta}(x, y)=u_{i}^{0}(x, y)+\eta u_{i}^{1}(x, y)+\eta^{2} u_{i}^{2}(x, y)+\cdots$

where $u_{i}^{\eta}$ is the exact value of the field quantity, $u^{0}, u^{1}, u^{2} \ldots$ are periodic with respect to the local coordinate $y$, which implies that each field quantity yield identical value on the opposite sides of the unit cell. The microscopic coordinates $y$ and the macroscopic coordinates $x$ can be related through

$y_{i}=\frac{x_{i}}{\eta}$

where $\eta$ is the magnification factor, which scales the size of the unit cell to the size of structure at macroscale. If $g^{\eta}=g^{\eta}(x, y)$ and $y$ depends on $x$, then the derivatives of the function with respect to $x$ can be written using the chain rule:

$\frac{\partial g^{\eta}}{\partial x_{i}}=\frac{\partial g}{\partial x_{i}}+\frac{1}{\eta} \frac{\partial g}{\partial y_{i}}$

According to Eqs. (A.3), we can obtain the small deformation strain tensor to be written as

$$
\begin{gathered}
\varepsilon_{k l}^{\eta}=\frac{1}{2}\left(\frac{\partial u_{k}^{\eta}}{\partial x_{l}^{\eta}}+\frac{\partial u_{l}^{\eta}}{\partial x_{k}^{\eta}}\right)=\frac{1}{2}\left(\frac{1}{\eta}\left(\frac{\partial u_{k}^{0}}{\partial y_{l}}+\frac{\partial u_{l}^{0}}{\partial y_{k}}\right)+\left(\frac{\partial u_{k}^{0}}{\partial x_{l}}+\frac{\partial u_{l}^{0}}{\partial x_{k}}+\frac{\partial u_{k}^{1}}{\partial y_{l}}+\frac{\partial u_{l}^{1}}{\partial y_{k}}\right)\right. \\
\left.+\eta\left(\frac{\partial u_{k}^{1}}{\partial x_{l}}+\frac{\partial u_{l}^{1}}{\partial x_{k}}+\frac{\partial u_{k}^{2}}{\partial y_{l}}+\frac{\partial u_{l}^{2}}{\partial y_{k}}\right)+\eta^{2}\left(\frac{\partial u_{k}^{2}}{\partial x_{l}}+\frac{\partial u_{l}^{2}}{\partial x_{k}}+\frac{\partial u_{k}^{3}}{\partial y_{l}}+\frac{\partial u_{l}^{3}}{\partial y_{k}}\right)+\cdots\right)
\end{gathered}
$$

The strain tensor can be rewritten as:

$\varepsilon_{k l}^{\eta}=\frac{1}{\eta} \varepsilon_{k l}^{-1}+\varepsilon_{k l}^{0}+\eta \varepsilon_{k l}^{1}+\eta^{2} \varepsilon_{k l}^{2}+\cdots$ where

$\varepsilon_{i j}^{-1}=\frac{1}{2}\left(\frac{\partial u_{i}^{0}}{\partial y_{j}}+\frac{\partial u_{j}^{0}}{\partial y_{i}}\right)$

$\varepsilon_{i j}^{0}=\frac{1}{2}\left(\frac{\partial u_{i}^{0}}{\partial x_{j}}+\frac{\partial u_{j}^{0}}{\partial x_{i}}\right)+\frac{1}{2}\left(\frac{\partial u_{i}^{1}}{\partial y_{j}}+\frac{\partial u_{j}^{1}}{\partial y_{i}}\right)$

$\varepsilon_{i j}^{1}=\frac{1}{2}\left(\frac{\partial u_{i}^{1}}{\partial x_{j}}+\frac{\partial u_{j}^{1}}{\partial x_{i}}\right)+\frac{1}{2}\left(\frac{\partial u_{i}^{2}}{\partial y_{j}}+\frac{\partial u_{j}^{2}}{\partial y_{i}}\right)$

Substituting the Eqs. (A.5) into the constitutive equation, we can obtain the asymptotic expansion of the stress field:

$\sigma_{k l}^{\eta}=\frac{1}{\eta} \sigma_{k l}^{-1}+\sigma_{k l}^{0}+\eta \sigma_{k l}^{1}+\eta^{2} \sigma_{k l}^{2}+\cdots$

Then, substituting the Eqs. (A.9) into the equilibrium equation, we can obtain:

$\frac{1}{\eta}\left[\frac{\partial \sigma_{i j}^{-1}}{\partial x_{j}}+\frac{1}{\eta} \frac{\partial \sigma_{i j}^{-1}}{\partial y_{j}}\right]+\left[\frac{\partial \sigma_{i j}^{0}}{\partial x_{j}}+\frac{1}{\eta} \frac{\partial \sigma_{i j}^{0}}{\partial y_{j}}\right]+\eta\left[\frac{\partial \sigma_{i j}^{1}}{\partial x_{j}}+\frac{1}{\eta} \frac{\partial \sigma_{i j}^{1}}{\partial y_{j}}\right]+\cdots+f_{i}=0$

By equating the terms with the same power of $\eta$, equations can be derived:

$\eta^{-2}: \frac{\partial \sigma_{i j}^{-1}}{\partial y_{j}}=\frac{\partial}{\partial y_{j}} C_{i j k l} \frac{\partial u_{k}^{0}}{\partial y_{l}}=0$

$\eta^{-1}: \frac{\partial \sigma_{i j}^{-1}}{\partial x_{j}}+\frac{\partial \sigma_{i j}^{0}}{\partial y_{j}}=\frac{\partial}{\partial x_{j}} C_{i j k l} \frac{\partial u_{k}^{0}}{\partial y_{l}}+\frac{\partial}{\partial y_{j}} C_{i j k l}\left(\frac{\partial u_{k}^{0}}{\partial y_{l}}+\frac{\partial u_{k}^{1}}{\partial y_{l}}\right)=0$

$\eta^{0}: \frac{\partial \sigma_{i j}^{0}}{\partial x_{j}}+\frac{\partial \sigma_{i j}^{1}}{\partial y_{j}}+f_{i}=\frac{\partial}{\partial x_{j}} C_{i j k l}\left(\frac{\partial u_{k}^{0}}{\partial y_{l}}+\frac{\partial u_{k}^{1}}{\partial y_{l}}\right)+\frac{\partial}{\partial y_{j}} C_{i j k l}\left(\frac{\partial u_{k}^{1}}{\partial y_{l}}+\frac{\partial u_{k}^{2}}{\partial y_{l}}\right)$

$+f_{i}=0$

$\eta^{1}: \frac{\partial \sigma_{i j}^{1}}{\partial x_{j}}+\frac{\partial \sigma_{i j}^{2}}{\partial y_{j}}=\frac{\partial}{\partial x_{j}} C_{i j k l}\left(\frac{\partial u_{k}^{1}}{\partial y_{l}}+\frac{\partial u_{k}^{2}}{\partial y_{l}}\right)+\frac{\partial}{\partial y_{j}} C_{i j k l}\left(\frac{\partial u_{k}^{2}}{\partial y_{l}}+\frac{\partial u_{k}^{3}}{\partial y_{l}}\right)=0$ 
For the positive definiteness of the elastic tensor $D_{i j k l}$, we can derived:

$$
\frac{\partial u_{i}^{0}}{\partial y_{j}}=0
$$

Eqs. (A.15) indicates that $u_{i}^{0}$ depend only on the macroscopic scale. It can be treated as the average value of the displacement field. Eqs. (A.4) can be rewritten as:

$$
\begin{aligned}
& \varepsilon_{k l}^{\eta}(\mathbf{u})=\frac{1}{2}\left(\frac{\partial u_{k}^{\eta}}{\partial x_{l}^{\eta}}+\frac{\partial u_{l}^{\eta}}{\partial x_{k}^{\eta}}\right) \\
& \quad=\frac{1}{2}\left[\left(\frac{\partial u_{k}^{0}}{\partial x_{l}}+\frac{\partial u_{l}^{0}}{\partial x_{k}}\right)+\left(\frac{\partial u_{k}^{1}}{\partial y_{l}}+\frac{\partial u_{l}^{1}}{\partial y_{k}}\right)+\eta\left(\frac{\partial u_{k}^{1}}{\partial x_{l}}+\frac{\partial u_{l}^{1}}{\partial x_{k}}\right)+\cdots\right]+\text { h.o.t. }
\end{aligned}
$$

where h.o.t. represents higher order terms. $u_{i}^{1}, u_{i}^{2}, \ldots$ are the microscopic displacement of different orders. Substituting Eqs. (A.15) in to the Eqs. (A.12), we can obtain:

$\frac{\partial}{\partial y_{j}} C_{i j k l}\left(\frac{\partial u_{k}^{0}}{\partial x_{l}}+\frac{\partial u_{k}^{1}}{\partial y_{l}}\right)=0$

The macroscopic displacement and the first order microscopic displacement are related in the Eqs. (A.17). If the macroscopic displacement is known, the first order microscopic displacement can be calculated through

$\frac{\partial}{\partial y_{j}}\left(C_{i j k l} \frac{\partial u_{k}^{1}}{\partial y_{l}}\right)=-\frac{\partial C_{i j k l}}{\partial y_{j}} \frac{\partial u_{k}^{0}}{\partial y_{l}}$

Generally, finite element method can be used to solve the function. For simplicity, eigenfunction, $\chi_{k}^{m n}\left(y_{i}\right)$, is introduced to relate the macroscopic displacement and microscopic displacement.

$u_{i}^{1}=\chi_{i}^{k l} \frac{\partial u_{k}^{0}}{\partial x_{l}}$

Then Eqs. (A.18) can be written as

$\frac{\partial}{\partial y_{j}}\left(C_{i j k l} \frac{\partial \chi_{k}^{m n}}{\partial y_{l}}\right)=-\frac{\partial C_{i j k l}}{\partial y_{j}}$.

The weak form of integration can be expressed as

$\int_{Y} \frac{\partial}{\partial y_{j}}\left(C_{i j k l} \frac{\partial \chi_{k}^{m n}}{\partial y_{l}}\right) v_{i} d Y=-\int_{Y} \frac{\partial C_{i j k l}}{\partial y_{j}} v_{j} d Y \forall v_{i} \in V_{Y}$

where $v$ is the virtual displacement.

Substituting Eqs. (A.19) into (A.7), we can obtain the macro strain tensor

$$
\begin{aligned}
\varepsilon_{i j}^{0} & =\frac{1}{2}\left(\frac{\partial u_{i}^{0}}{\partial x_{j}}+\frac{\partial u_{j}^{0}}{\partial x_{i}}+\frac{\partial u_{i}^{1}}{\partial y_{j}}+\frac{\partial u_{j}^{1}}{\partial y_{i}}\right) \\
& =\frac{1}{2}\left(\frac{\partial u_{i}^{0}}{\partial x_{j}}+\frac{\partial u_{j}^{0}}{\partial x_{i}}+\frac{\partial \chi_{i}^{m n}}{\partial y_{j}} \frac{\partial u_{m}^{0}}{\partial x_{n}}+\frac{\partial \chi_{j}^{m n}}{\partial y_{i}} \frac{\partial u_{m}^{0}}{\partial x_{n}}\right) . \\
& =\left(\delta_{i m} \delta_{j n}+\frac{\partial \chi_{i}^{m n}}{\partial y_{j}}\right) \frac{\partial u_{m}^{0}}{\partial x_{n}}
\end{aligned}
$$

The corresponding macro stress tensor can be expressed as

$\sigma_{i j}^{0}=C_{i j k l} \varepsilon_{k l}^{0}=C_{i j k l}\left(\delta_{k m} \delta_{l n}+\frac{\partial \chi_{k}^{m n}}{\partial y_{l}}\right) \frac{\partial u_{m}^{0}}{\partial x_{n}}$.

Integrating Eqs. (A.13), one concludes that

$\frac{\partial}{\partial x_{j}} \int_{V} \sigma_{i j}^{0} d V+\int_{V} \frac{\partial \sigma_{i j}^{1}}{\partial y_{j}} d V+\int_{V} f_{i} d V=0$

Because $\sigma_{i j}^{1}$ is Y-periodic, Eqs. (A.24) can be written as

$\frac{\partial}{\partial x_{j}} \int_{V} \sigma_{i j}^{0} d V+\int_{V} f_{i} d V=0$

Then, substituting Eqs. (A.23) into Eqs. (A.25), we can conclude the homogenizedequilibrium equation:

$\frac{\partial}{\partial x_{j}} \bar{D}_{i j k l} \frac{\partial u_{m}^{0}}{\partial x_{n}}+f_{i}=0$ where $\bar{D}_{i j k l}$ is the homogenized elastic tensor.

$\bar{D}_{i j m n}=\frac{1}{V} \int_{V} D_{i j k l}\left(\delta_{k m} \delta_{\mathrm{ln}}+\frac{\partial \chi_{k}^{m n}}{\partial y_{l}}\right) d V$

For the calculation of $\chi_{k}^{m n}\left(y_{i}\right)$, Gauss theorem is applied on the two sides of Eqs. (A.21), the final equilibrium equation is obtained

$\int_{Y} D_{i j k l} \frac{\partial \chi_{k}^{m n}}{\partial y_{l}} \frac{\partial v_{i}}{\partial y_{j}} d Y=-\int_{S} D_{i j m n} n_{j} v_{i} d S$

where $S$ is the surface of the lattice, $n_{j}$ is the $j$ thcomponent of the lattice surface outer normal. The right side of Eqs. (A.28) can be treated as the surface traction, $T_{m n}$. The $\chi_{k}^{m n}$ can be obtained by the calculation of displacement field under different surface traction boundary condition in ABAQUS.

\section{Appendix B}

\section{B.1 Relative density of the octet-truss structures}

The relative density of the octet-truss given in Eqs. (2) is in the case of no taper. It can be derived through analysis of the exact geometry of a single beam, as shown in Fig. B1(a). For a single beam, the apex angles are different in the cross section of the rectangular pyramids. To simplify the calculation, we can assume that the apex angle is the same and then calculate the average volume of two beams. The total volume can be divided into three parts:

(1) average volume oftwo cylinders with radius $R$ and the length $\operatorname{are} L-2 R$ and $L-2 \sqrt{2} R$,

(2) (2)average volume of two rectangular pyramidswith bottom side length $\sqrt{2} R$ and the height are Rand $\sqrt{2} R$,

(3) average volume ofeight cylindrical wedges of radius $R$ and the height are $(\sqrt{2}-1) R$ or $(2-\sqrt{2}) R$.

The volume of a single beam can be calculated by

$V_{o t}=V_{o t-1}+V_{o t-2}-V_{o t-3}$

where $V_{o t-1}$ is the volume of the cylinder and is given by

$V_{o t-1}=\frac{1}{2} \pi R^{2}((L-2 R)+(L-2 \sqrt{2} R))$

$V_{o t-2}$ is the volume of the two rectangular pyramids and is given by

$V_{o t-2}=2\left[\frac{1}{2}\left(\frac{2}{3} R^{3}+\frac{2 \sqrt{2}}{3} R^{3}\right)\right]$

$V_{o t-3}$ is the volume of the eight cylindrical wedges and is given by

$V_{o t-3}=8\left[\frac{1}{2}\left(\frac{2-\sqrt{2}}{\sqrt{2}-1}\left(\frac{5 \sqrt{2}}{12} R^{3}-\frac{\sqrt{2}}{8} \pi R^{3}\right)+2\left(\frac{5 \sqrt{2}}{12} R^{3}-\frac{\sqrt{2}}{8} \pi R^{3}\right)\right)\right]$

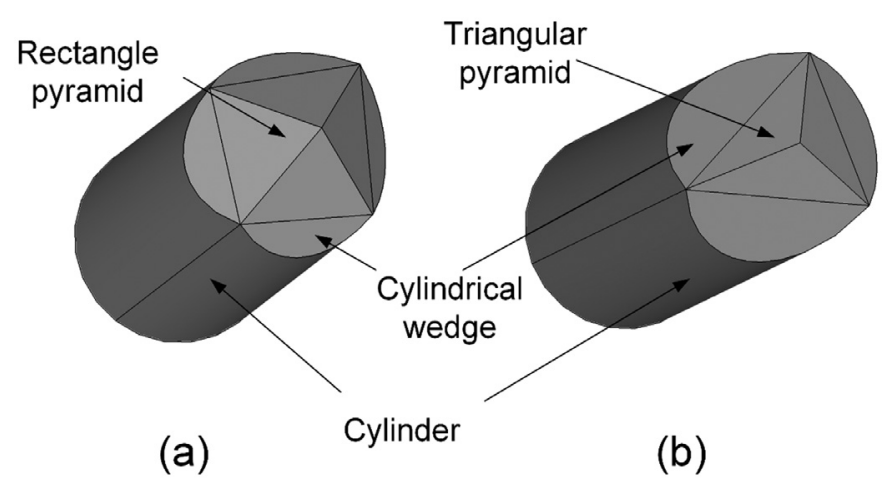

Fig. B1. 3D view of single beam of (a) octet-truss lattice structure; (b) truncatedoctahedron lattice structure for relative density calculation. 
Then the total volume is obtained

$V_{o t}=\pi R^{2} L-\frac{8 R^{3}}{3(\sqrt{2}-1)}$,

and then, considering there are 24 integral beams in unit cell, the relative density can be calculated

$\rho_{t o t}^{*}=\frac{24 V_{o t}}{(\sqrt{2} L)^{3}}=6 \sqrt{2} \pi\left(\frac{R}{L}\right)^{2}-\frac{32}{2-\sqrt{2}}\left(\frac{R}{L}\right)^{3}$

For tapped octet-truss structure, the two ends of the beam are enhanced with the radius $\sqrt{2} R$ and the length is $4 \sqrt{2} R / 3$. Then the total volume of the single beam can be given by

$V_{t o t}=\pi R^{2} L f+\pi R^{3}(1+\sqrt{2})(1-f)-\frac{8(\sqrt{2}+1)}{3} R^{3}$

where $f=1.1683$.

The corresponding relative density can be calculated

$\rho_{\text {tot }}^{*}=6 \sqrt{2} \pi\left(\frac{R}{L}\right)^{2} f+\pi\left(\frac{R}{L}\right)^{3}(6 \sqrt{2}+12)(1-f)-\frac{32}{2-\sqrt{2}}\left(\frac{R}{L}\right)^{3}$

\section{B.2 Relative density of the truncated-octahedron structures}

The relative density of the truncated-octahedron can also be derived through analysis of the exact geometry of a single beam, as shown in Fig. B1(b). For a single beam, the apex is triangular pyramid. The total volume can be divided into three parts:

(1) volume of cylinder with radius $R$ and the length is $L-\frac{2}{3} R$,

(2) volume of two triangular pyramids with bottom area $\frac{8 \sqrt{2}}{9} R^{2}$ and height $\frac{1}{3} R$,

(3) volume of six cylindrical wedges of radius $R$ and the height are $\frac{2}{3} R$ and $\frac{\sqrt{3}-1}{3} R$.

The volume of single ambient beam in truncated-octahedron structure can be calculated by

$V_{t o}=V_{t o-1}+V_{t o-2}-V_{t o-3}$

where $V_{t o-1}$ is the volume of the cylinder and is given by

$V_{t o-1}=\pi R^{2}\left(L-\frac{2}{3} R\right)$

$V_{t o-2}$ is the volume of the two triangular pyramids and is given by

$V_{t o-2}=\frac{8 \sqrt{2}}{81} R^{3}$

$V_{t o-3}$ is the volume of the six cylindrical wedges and is given by

$V_{t o-3}=\frac{208 \sqrt{2}}{81} R^{3}-\frac{2}{3} R^{3}\left(2 \theta_{1}+\theta_{2}+\sin 2 \theta_{1}+\frac{1}{2} \sin 2 \theta_{2}\right)$

where $\theta_{1}=\arcsin (\sqrt{6} / 3)$ and $\theta_{2}=\arcsin (\sqrt{8} / 3)$.

Then the total volume is obtained

$V_{t o}=\pi R^{2} L-\frac{200 \sqrt{2}}{81} R^{3}+\frac{2}{3} R^{3}\left[2 \theta_{1}+\theta_{2}+\sin 2 \theta_{1}+\frac{1}{2} \sin 2 \theta_{2}-\pi\right]$

and then, considering there are 24 integral beams in unit cell, the relative density can be calculated

$$
\begin{aligned}
\rho_{t o}^{*}= & \frac{24 V}{(2 \sqrt{2} L)^{3}}=\frac{3}{4} \sqrt{2} \pi\left(\frac{R}{L}\right)^{2}-\frac{100}{27}\left(\frac{R}{L}\right)^{3} \\
& +\frac{\sqrt{2}}{2}\left(\frac{R}{L}\right)^{3}\left[2 \theta_{1}+\theta_{2}+\sin 2 \theta_{1}+\frac{1}{2} \sin 2 \theta_{2}-\pi\right]
\end{aligned}
$$

For tapped truncated-octahedron structure, the two ends of the beam are enhanced with the radius $7 R / 5$ and the length is $9 R / 8$. The total volume of the single beam is given by

$V_{t t o}=\pi R^{2} L f-\frac{200 \sqrt{2}}{81} R^{3}+\frac{2}{3} R^{3}\left(2 \theta_{1}+\theta_{2}+\sin 2 \theta_{1}+\frac{1}{2} \sin 2 \theta_{2}-\pi f\right)$

where $f=1.3732$.

The corresponding relative density can be calculated

$$
\begin{aligned}
\rho_{\text {tto }}^{*}= & \frac{3}{4} \sqrt{2} \pi\left(\frac{R}{L}\right)^{2} f-\frac{100}{27}\left(\frac{R}{L}\right)^{3} \\
& +\frac{\sqrt{2}}{2}\left(\frac{R}{L}\right)^{3}\left(2 \theta_{1}+\theta_{2}+\sin 2 \theta_{1}+\frac{1}{2} \sin 2 \theta_{2}-\pi f\right)
\end{aligned}
$$

\section{References}

[1] Queheillalt DT, Murty Y, Wadley HNG. Mechanical properties of an extruded pyramidal lattice truss sandwich structure. Scripta Mater 2008;58(1):76-9. doi:10.1016/j.scriptamat.2007.08.041.

[2] Tancogne-Dejean T, Spierings AB, Mohr D. Additively-manufactured metallic microlattice materials for high specific energy absorption under static and dynamic loading. Acta Mater 2016;116:14-28. doi:10.1016/j.actamat.2016.05.054.

[3] Kaur M, Yun TG, Han SM, Thomas EL, Kim WS. 3D printed stretching-dominated micro-trusses. Mater Design 2017;134:272-80. doi:10.1016/j.matdes.2017.08.061.

[4] Qi D, Yu H, Hu W, He C, Wu W, Ma Y. Bandgap and wave attenuation mechanisms of innovative reentrant and anti-chiral hybrid auxetic metastructure. Extreme Mech. Lett. 2019;28:58-68. doi:10.1016/j.eml.2019.02.005.

[5] Wu W, Hu W, Qian G, Liao H, Xu X, Berto F. Mechanical design and multifunctional applications of chiral mechanical metamaterials: a review. Mater. Design 2019;180:107950. doi:10.1016/j.matdes.2019.107950.

[6] Wu W, Qi D, Liao H, Qian G, Geng L, Niu Y, et al. Deformation mechanism of innovative 3D chiral metamaterials. Sci. Rep. 2018;8(1):12575. doi:10.1038/s41598-018-30737-7.

[7] Wu W, Song X, Liang J, Xia R, Qian G, Fang D. Mechanical properties of anti-tetrachiral auxetic stents. Compos Struct 2018;185:381-92. doi:10.1016/j.compstruct.2017.11.048.

[8] Wu W, Tao Y, Xia Y, Chen J, Lei H, Sun L, et al. Mechanical properties of hierarchical anti-tetrachiral metastructures. Extreme Mech. Lett. 2017;16:18-32. doi:10.1016/j.eml.2017.08.004.

[9] Yu H, Wu W, Zhang J, Chen J, Liao H, Fang D. Drastic tailorable thermal expansion chiral planar and cylindrical shell structures explored with finite element simulation. Compos Struct 2019;210:327-38. doi:10.1016/j.compstruct.2018.11.043.

[10] Xu H, Pasini D. Structurally efficient three-dimensional metamaterials with controllable thermal expansion. Sci. Rep. 2016;6:34924. doi:10.1038/srep34924.

[11] Elsayed MSA, Pasini D. Multiscale structural design of columns made of regular octet-truss lattice material. Int J Solids Struct 2010;47(14):1764-74. doi:10.1016/j.ijsolstr.2010.03.003.

[12] Xiao D, Dong Z, Li Y, Wu W, Fang D. Compression behavior of the graded metallic auxetic reentrant honeycomb: experiment and finite element analysis. Mater Sci Eng A 2019;758:163-71. https://doi.org/10.1016/j.msea.2019.04.116.

[13] Chen XY, Tan HF. An effective length model for octet lattice. Int J Mech Sci 2018;140:279-87. doi:10.1016/j.ijmecsci.2018.03.016.

[14] Xu S, Shen J, Zhou S, Huang X, Xie YM. Design of lattice structures with controlled anisotropy. Mater Design 2016;93:443-7. doi:10.1016/j.matdes.2016.01.007.

[15] Meza LR, Phlipot GP, Portela CM, Maggi A, Montemayor LC, Comella A, et al. Reexamining the mechanical property space of three-dimensional lattice architectures. Acta Mater 2017;140:424-32. doi:10.1016/j.actamat.2017.08.052.

[16] Tancogne-Dejean T, Mohr D. Stiffness and specific energy absorption of additivelymanufactured metallic BCC metamaterials composed of tapered beams. Int J Mech Sci 2018;141:101-16. doi:10.1016/j.ijmecsci.2018.03.027.

[17] Amani Y, Dancette S, Delroisse P, Simar A, Maire E. Compression behavior of lattice structures produced by selective laser melting: X-ray tomography based experimental and finite element approaches. Acta Mater 2018;159:395-407. doi:10.1016/j.actamat.2018.08.030.

[18] Liu L, Kamm P, García-Moreno F, Banhart J, Pasini D. Elastic and failure response of imperfect three-dimensional metallic lattices: the role of geometric defects induced by selective laser melting. J Mech Phys Solids 2017;107:160-84. doi:10.1016/j.jmps.2017.07.003.

[19] Abdelhamid M, Czekanski A. Impact of the lattice angle on the effective properties of the octet-truss lattice structure. J Eng Mater Technol 2018;140(4) 041010-041010041011. doi:10.1115/1.4040409.

[20] Portela CM, Greer JR, Kochmann DM. Impact of node geometry on the effective stiffness of non-slender three-dimensional truss lattice architectures. Extrem Mech Lett 2018;22:138-48. doi:10.1016/j.eml.2018.06.004.

[21] He Z, Wang F, Zhu Y, Wu H, Park HS. Mechanical properties of copper octet-truss nanolattices. J Mech Phys Solids 2017;101:133-49. doi:10.1016/j.jmps.2017.01.019.

[22] Carlton HD, Lind J, Messner MC, Volkoff-Shoemaker NA, Barnard HS, Barton NR, et al. Mapping local deformation behavior in single cell metal lattice structures. Acta Mater 2017;129:239-50. doi:10.1016/j.actamat.2017.02.023. 
[23] Sugimura Y. Mechanical response of single-layer tetrahedral trusses under shear loading. Mech Mater 2004;36(8):715-21. doi:10.1016/j.mechmat.2003.05.002.

[24] Dong L, Deshpande V, Wadley H. Mechanical response of Ti-6Al-4 V octet-truss lattice structures. Int $\mathrm{J}$ Solids Struct 2015;60-61:107-24. doi:10.1016/j.ijsolstr.2015.02.020.

[25] Song J, Gao L, Cao K, Zhang H, Xu S, Jiang C, et al. Metal-coated hybrid meso-lattice composites and their mechanical characterizations. Compos Struct 2018;203:75063. doi:10.1016/j.compstruct.2018.07.074.

[26] Wu Q, Vaziri A, Asl ME, Ghosh R, Gao Y, Wei X, et al. Lattice materials with pyramidal hierarchy: systematic analysis and three dimensional failure mechanism maps. J. Mech. Phys. Solids 2019;125:112-44. doi:10.1016/j.jmps.2018.12.006.

[27] Fan HL, Jin FN, Fang DN. Nonlinear mechanical properties of lattice truss materials. Mater Design 2009;30(3):511-17. doi:10.1016/j.matdes.2008.05.061.

[28] Niu J, Choo HL, Sun W, Mok SH. Numerical study on load-bearing capabilities of beam-like lattice structures with three different unit cells. Int J Mech Mater Des 2018;14(3):443-60. doi:10.1007/s10999-017-9384-3.

[29] Xiao Z, Yang Y, Xiao R, Bai Y, Song C, Wang D. Evaluation of topology-optimized lattice structures manufactured via selective laser melting. Mater Design 2018;143:2737. doi:10.1016/j.matdes.2018.01.023.

[30] Sercombe TB, Xu X, Challis VJ, Green R, Yue S, Zhang Z, et al. Failure modes in high strength and stiffness to weight scaffolds produced by selective laser melting. Mater Design 2015;67:501-8. doi:10.1016/j.matdes.2014.10.063.

[31] Messner MC. Optimal lattice-structured materials. J Mech Phys Solids 2016;96:16283. doi:10.1016/j.jmps.2016.07.010.

[32] Ushijima K, Cantwell WJ, Chen DH. Prediction of the mechanical properties of microlattice structures subjected to multi-axial loading. Int J Mech Sci 2013;68:47-55. doi:10.1016/j.ijmecsci.2012.12.017.

[33] Dong L, Wadley H. Shear response of carbon fiber composite octettruss lattice structures. Compos Part A Appl Sci Manuf 2016;81:182-92. doi:10.1016/j.compositesa.2015.11.015

[34] O'Masta MR, Dong L, St-Pierre L, Wadley HNG, Deshpande VS. The fracture toughness of octet-truss lattices. J Mech Phys Solids 2017;98:271-89. doi:10.1016/j.jmps.2016.09.009.

[35] Zhang M, Yang Z, Lu Z, Liao B, He X. Effective elastic properties and initial yield surfaces of two 3D lattice structures. Int J Mech Sci 2018;138-139:146-58. doi:10.1016/j.ijmecsci.2018.02.008.

[36] Li X, Xiong J, Ma L, Wu L, Yan X. Effect of vacuum thermal cycling on the compression and shear performance of composite sandwich structures containing pyramidal truss cores. Compos Sci Technol 2018;158:67-78. doi:10.1016/j.compscitech.2018.01.042.

[37] Latture RM, Rodriguez RX, Holmes LR, Zok FW. Effects of nodal fillets and external boundaries on compressive response of an octet truss. Acta Mater 2018;149:78-87. doi:10.1016/j.actamat.2017.12.060.

[38] Tancogne-Dejean T, Mohr D. Elastically-isotropic truss lattice materials of reduced plastic anisotropy. Int J Solids Struct 2018;138:24-39. doi:10.1016/j.ijsolstr.2017.12.025.

[39] Berger JB, Wadley HNG, McMeeking RM. Mechanical metamaterials at the theoretical limit of isotropic elastic stiffness. Nature 2017;543:533. doi:10.1038/nature21075.
[40] Tancogne-Dejean T, Mohr D. Elastically-isotropic elementary cubic lattices composed of tailored hollow beams. Extreme Mech Lett 2018;22:13-18. doi:10.1016/j.eml.2018.04.005.

[41] Feng L-J, Wu L-Z, Yu G-C. An hourglass truss lattice structure and its mechanical performances. Mater Design 2016;99:581-91. doi:10.1016/j.matdes.2016.03.100.

[42] Kooistra GW, Deshpande VS, Wadley HNG. Compressive behavior of age hardenable tetrahedral lattice truss structures made from aluminium. Acta Mater 2004;52(14):4229-37. doi:10.1016/j.actamat.2004.05.039.

[43] Li C, Lei H, Liu Y, Zhang X, Xiong J, Zhou H, et al. Crushing behavior of multilayer metal lattice panel fabricated by selective laser melting. Int J Mech Sci 2018;145:389-99. doi:10.1016/j.ijmecsci.2018.07.029.

[44] Zhu S, Ma L, Wang B, Hu J, Zhou Z. Lattice materials composed by curved struts exhibit adjustable macroscopic stress-strain curves. Mater Today Commun 2018;14:273-81. doi:10.1016/j.mtcomm.2018.01.017.

[45] Beharic A, Rodriguez Egui R, Yang L. Drop-weight impact characteristics of additively manufactured sandwich structures with different cellular designs. Mater Design 2018;145:122-34. doi:10.1016/j.matdes.2018.02.066.

[46] Deshpande VS, Fleck NA, Ashby MF. Effective properties of the octet-truss lattice material. J Mech Phys Solids 2001;49(8):1747-69. doi:10.1016/S0022-5096(01)00010-2.

[47] Geng L, Wu W, Sun L, Fang D. Damage characterizations and simulation of selective laser melting fabricated 3D re-entrant lattices based on in-situ CT testing and geometric reconstruction. Int J Mech Sci 2019;157-158:231-42. doi:10.1016/j.ijmecsci.2019.04.054.

[48] Wang Z, Wu W, Qian G, Sun L, Li X, Correia JAFO. In-situ SEM investigation on fatigue behaviors of additive manufactured Al-Si10-Mg alloy at elevated temperature. Eng Fract Mech 2019;214:149-63. doi:10.1016/j.engfracmech.2019.03.040.

[49] Hassani B, Hinton E. A review of homogenization and topology opimization II-analytical and numerical solution of homogenization equations. Comput Struct 1998;69(6):719-38. doi:10.1016/S0045-7949(98)00132-1.

[50] Arabnejad S, Pasini D. Mechanical properties of lattice materials via asymptotic homogenization and comparison with alternative homogenization methods. Int J Mech Sci 2013;77:249-62. doi:10.1016/j.ijmecsci.2013.10.003.

[51] Andrianov IV, Bolshakov VI, Danishevs, kyy VV, Weichert D. Higher order asymptotic homogenization and wave propagation in periodic composite materials. Proc Roy Soc A Math Phys Eng Sci 2008;464(2093):1181. doi:10.1098/rspa.2007.0267.

[52] Kalamkarov AL, Andrianov IV, Danishevs'kyy VV. Asymptotic homogenization of composite materials and structures. Appl Mech Rev 2009;62(3) 030802-030802030820. doi:10.1115/1.3090830.

[53] Cai Y, Xu L, Cheng G. Novel numerical implementation of asymptotic homogenization method for periodic plate structures. Int J Solids Struct 2014;51(1):284-92. doi:10.1016/j.ijsolstr.2013.10.003.

[54] Gaillac R, Pullumbi P, Coudert F-X. ELATE: an open-source online application for analysis and visualization of elastic tensors. J Phys Condens Matter 2016;28(27):275201. doi:10.1088/0953-8984/28/27/275201. 